\title{
CARACTERÍSTICAS AMBIENTALES Y ALGAS NOCIVAS EN UN ÁREA DE PRODUCCIÓN DE MOLUSCOS BIVALVOS DEL CANAL BEAGLE, ARGENTINA
}

\author{
Hugo Benavides ${ }^{1}$, Nora G. Montoya ${ }^{1}$, Mario Carignan ${ }^{1}$ y Carlos Luizón ${ }^{2}$ \\ ${ }^{1}$ Instituto Nacional de Investigación y Desarrollo Pesquero (INIDEP), \\ Paseo Victoria Ocampo No 1, Escollera Norte, B7602HSA - Mar del Plata, Argentina \\ correo electrónico: hbenavid@inidep.edu.ar \\ ${ }^{2}$ Universidad Tecnológica Nacional, Facultad Regional Tierra del Fuego, \\ Islas Malvinas 1650, V9420AIW - Río Grande, Argentina
}

\begin{abstract}
RESUMEN. En diciembre de 2013 se realizó un estudio para conocer las especies de microalgas tóxicas presentes en el área, las distintas toxinas que producen y los factores ambientales que condicionan la distribución de las comunidades de fitoplancton y su estructura. Se observó una alteración en la estructura de la comunidad fitoplantónica respecto al mismo período de años anteriores que indicó un retraso en las primeras etapas de la sucesión clásica. Dicho retraso influyó en el patrón anual de formación de floraciones de especies tóxicas en el canal y, en consecuencia, en los niveles de toxicidad de los bivalvos que normalmente se registran en esa época. El análisis de HPLC mostró escaso número de toxinas paralizantes de moluscos que coincidió con una muy baja concentración de células tóxicas. En las muestras analizadas se detectaron principalmente gonyautoxinas, sin presencia de toxina amnésica de moluscos. Los resultados aportan un conocimiento más detallado del perfil tóxico de los bivalvos de interés comercial en el área, que complementa el programa de monitoreo en curso. El análisis de los parámetros ambientales mostró que el sector interior de Bahía A. Brown es un área con circulación restringida que puede funcionar como reservorio potencial de los estadios bentónicos de resistencia de algunos dinoflagelados, proveyendo un inóculo inicial para la formación de floraciones o de eventos de toxicidad locales. La ausencia de floraciones de especies tóxicas y el bajo nivel de toxicidad de los moluscos del área en ese período se asociaron con las inusuales condiciones climáticas que se registraron en la región durante los meses previos, lo cual advierte sobre la necesidad de contar con un registro continuo de las variables meteorológicas para tratar de predecir la ocurrencia de floraciones de algas nocivas en el canal.
\end{abstract}

Palabras clave: Canal Beagle, características ambientales, microalgas nocivas, perfil de toxinas.

\section{ENVIRONMENTAL FEATURES AND HARMFUL ALGAE IN AN AREA OF BIVALVE SHELLFISH PRODUCTION OF THE BEAGLE CHANNEL, ARGENTINA}

\footnotetext{
ABSTRACT. In December 2013 a study was carried out with the objective of knowing the toxic microalgae species present in the area, the different toxins they produce and the environmental factors that condition the distribution of phytoplankton communities and their structure. An alteration in the phytoplankton community structure with respect to the same period of previous years indicated a delay in the early stages of the classic succession. Said delay influenced the annual pattern of harmful species blooms formation in the channel and, consequently, the levels of toxicity of the bivalves normally recorded at that time of the year. The HPLC analysis showed a scarce number of paralytic shellfish toxins that coincided with a very low concentration of toxic cells. In the samples analyzed, the toxins detected were mainly gonyautoxins, with no amnesic shellfish toxin. The results contribute a more detailed knowledge of the toxic profile of bivalves of commercial interest in the area that complements the ongoing monitoring program. The analysis
} 
of the environmental parameters showed that the inner sector of A. Brown Bay is an area with restricted circulation that may function as a potential reservoir for the benthic resistance stages of some dinoflagellates, providing an initial inoculum for blooms or local toxicity events formation. The absence of toxic species blooms and the low level of toxicity of the molluscs in the area in that period were associated to unusual climatic conditions registered in the region during the previous months, event that warns about the need to count on a continuous record of the meteorological variables in order to try to predict the occurrence of harmful algae blooms in the channel.

Key words: Beagle Channel, environmental features, harmful microalgae, toxins profile.

\section{INTRODUCCIÓN}

Las floraciones de dinoflagelados tóxicos ocurridas en Canal Beagle en los últimos años han causado grandes perjuicios económicos en la pesquería de moluscos bivalvos, obligando a imponer vedas en su recolección y comercialización. En esta región se explota comercialmente el mejillón (Mytilus chilensis), obtenido por recolección o cultivo a una escala artesanal. Se estimó que el $31,9 \%$ de la producción corresponde al mejillón de cultivo extraído en la zona de Puerto Almanza, en la Bahía A. Brown (Bertolotti et al. 2014), siendo considerada el área de producción más austral del mundo. Las posibilidades para el crecimiento y desarrollo regional de la actividad presentan algunas dificultades relacionadas a la alta frecuencia de eventos tóxicos causados por el florecimiento de microalgas toxicas, conocidos como "marea roja", los que originan vedas que pueden inmovilizar la producción por largos períodos y que habitualmente coinciden con el momento de recolección de la cosecha (Álvarez 2012; Bertolotti et al. 2014).

Las toxinas de mayor riesgo registradas en el Canal Beagle son las denominadas "Toxinas Paralizantes de los Moluscos" (TPM), un grupo de neurotoxinas originadas por dinoflagelados del género Alexandrium, que son acumuladas en los moluscos bivalvos por filtración. Aunque los florecimientos de Alexandrium catenella fueron registrados en la región de los fiordos chilenos en 1972 (Guzmán y Campodónico 1975; Guzmán y Lembeye 1975), no se detectaron evidencias de toxicidad en las costas de la Provincia de Tierra del Fuego, Antártida e Islas del Atlántico Sur hasta fines de 1991. En esta temporada se produjo una intensa marea roja provocada por $A$. catenella, registrándose niveles de toxicidad extremadamente elevados (127.200 $\mu \mathrm{g}$ de STX eq. $\left.100 \mathrm{~g}^{-1}\right)$ (Benavides et al. 1995). Esta marea roja produjo un gran impacto social y económico en la región, causando varias muertes humanas, numerosas intoxicaciones y mortandad de organismos marinos. A partir de ese suceso, el florecimiento de microalgas tóxica en la costa argentina del canal se repitió anualmente con mayor o menor intensidad. Otras especies nocivas, como algunos dinoflagelados del Género Dinophysis, han sido detectadas posteriormente en la región, ocasionando vedas por "Toxina Diarreica de Moluscos" (TDM) (Almandoz et al. 2011; Álvarez 2012; Goya y Maldonado 2014). Recientemente se detectó en el canal la presencia de Toxina Amnésica de Moluscos (TAM) (Krock et al. 2015; Almandoz et al. 2017) producida por algunas especies de diatomeas del Género Pseudo-nitzschia, aunque aún no se han identificado las especies responsables. También en esta región se han detectado, por primera vez en el país, toxinas del grupo "espirólido" en mejillones y en muestras naturales de fitoplancton (Montoya et al. 2012; Almandoz et al. 2014). Los espirólidos son un grupo de toxinas marinas producidas por algunos dinoflagelados y conocidas como "toxinas de rápida acción" por su inmediato efecto letal en los bioensayos, siendo $A$. ostenfeldii la especie identificada como productora en el Canal Beagle. Esta especie fue también asociada a la producción de TPM, presentando una gran variabilidad en la 
producción de uno u otro grupo de toxinas de acuerdo con su localización geográfica (Cembella et al. 2000; Touzet et al. 2008; Kremp et al. 2009).

Entre los días 7 y 20 de diciembre de 2013, se realizó una campaña de investigación en el área de Canal Beagle con el objetivo de identificar las especies tóxicas o potencialmente tóxicas presentes en el plancton y los factores ambientales asociados a su desarrollo, conocer su perfil de toxinas y las transformaciones que estas experimentan al ser metabolizadas por organismos de diferentes niveles tróficos. También se aislaron especies de microalgas nocivas, cuyos cultivos fueron empleados para realizar estudios autoecológicos y que permitieron analizar en detalle las características fisiológicas y químicas de algunas de las especies que periódicamente ocasionan eventos tóxicos en Canal Beagle.

\section{MATERIALES Y MÉTODOS}

\section{Área de estudio}

El estudio se realizó en un área del sector argentino del Canal Beagle correspondiente a la

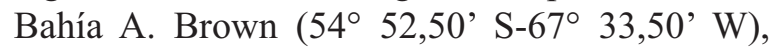
principal área de cultivo y extracción comercial de moluscos bivalvos en la región (Figura 1).

Se realizaron 14 estaciones de muestreo en el área de Bahía A. Brown y su sistema de drenaje (Figura 1). Las estaciones 5, 6 y 7 se ubicaron en la boca de la bahía, de la cuales la estación 6 fue realizada en las proximidades de una balsa de cultivo de mejillones fijada en el sector central de la bahía. Las estaciones 8,9 y 10 se ubicaron en el sector central y 11, 12 y 13 en el sector interno. También se realizaron dos estaciones entre la Isla Upú y la Isla Waru (estaciones 17 y 18) y tres en el Paso R. Guaraní medio (estaciones 14, 15 y 16). Con el objetivo de evaluar la variación de los parámetros ambientales en el corto plazo y su relación con la estructura del fitoplancton, el muestreo realizado previamente en las estaciones $6,11,14$ y 17 fue repetido después de un período de $7 \mathrm{~d}$ (estaciones 22, 23, 24 y 25).

\section{Metodología}

En cada estación de muestro se estableció la posición geográfica (GPS), se registraron las variables ambientales, se tomaron muestras de fitoplancton cuantitativas y de red, y se colectaron muestras de mejillones en los bancos naturales o de la balsa de cultivo. En las estaciones 4, 6, 14,22 y 25 se obtuvieron muestras de zooplancton y de fitoplancton por fracciones de tamaño para análisis de toxinas; y muestras para análisis de nutrientes y pigmentos fotosintéticos. La obtención de las muestras y medición de parámetros ambientales fueron realizadas empleando una embarcación semirígida.

\section{Parámetros ambientales}

Se registraron perfiles verticales de temperatura y conductividad del agua mediante un CTD autocontenido Citadel-NV, empleando una frecuencia de 25 muestras $\mathrm{s}^{-1}$ y una velocidad de descenso aproximada de $50 \mathrm{~cm} \mathrm{seg}^{-1}$. Se tomaron muestras de $250 \mathrm{~mL}$ de agua de mar para la determinación de salinidad, con el fin de calibrar las mediciones realizadas en los perfiles verticales de CTD.

Se tomaron muestras de agua superficial para análisis de pigmentos y de nutrientes en la entrada de Bahía A. Brown y Paso R. Guaraní medio. Para el análisis de pigmentos, muestras de 1,5 1 de agua fueron previamente tamizadas con una malla de $80 \mu \mathrm{m}$ y posteriormente filtradas por filtros de fibra de vidrio Wathman GF/F. Los filtros se almacenaron a $-20{ }^{\circ} \mathrm{C}$ para su posterior análisis en laboratorio por HPLC (Zapata et al. 2000). Para el análisis de nutrientes, $10 \mathrm{~mL}$ de cada muestra fueron filtrados por filtros de fibra de vidrio Wathman $\mathrm{GF} / \mathrm{F}$ y almacenados a $-20{ }^{\circ} \mathrm{C}$. El análisis de nutrientes (fosfato, silicato, nitrato $\mathrm{y}$ 

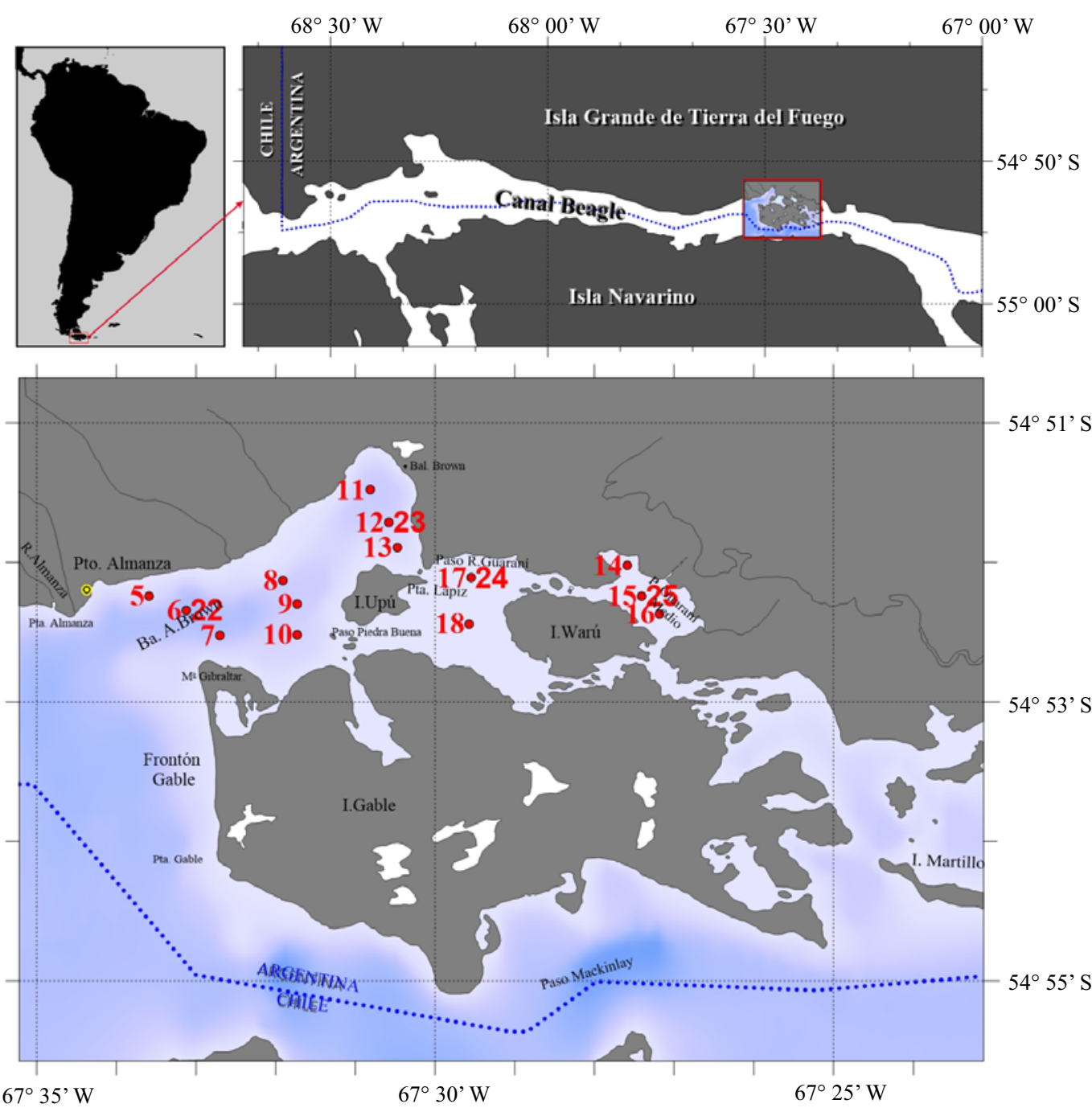

Figura 1. Ubicación geográfica de las estaciones de muestreo realizadas en Bahía A. Brown el 10 de diciembre (estaciones 5 a 18) y 17 de diciembre (estaciones 22 a 25).

Figure 1. Geographical location of sampling stations carried out in A. Brown Bay on December $10^{\text {th }}$ (stations 5 through 18) and December $17^{\text {th }}$ (stations 22 through 25).

nitrito) se realizó mediante un autoanalizador Technicon TA II (Grasshoff y Ehrhardt 1983; Hydes et al. 2010).

\section{Información climatológica}

El análisis de las condiciones climáticas fue realizado empleando los registros atmosféricos obtenidos por la estación meteorológica Ushuaia
Aerodrome 879380 (SAWH) ubicada en el aeropuerto internacional de la ciudad de Ushuaia (SMN 2013).

\section{Análisis de toxinas}

Para el análisis de toxinas en bivalvos, se colectaron muestras de aproximadamente $2 \mathrm{~kg}$ de mejillón (M. chilensis) y/o cholga (Aulacomya 
atra) de los bancos naturales próximos a las estaciones de toma de muestras de fitoplancton y de la balsa de cultivo situada en la entrada de Bahía A. Brown. Con el objetivo de estudiar la evolución temporal de la toxicidad, se analizaron muestras de mejillones y cholgas correspondientes al período julio-diciembre de 2013. Las muestras para la determinación de TPM fueron analizadas con el método oficial de bioensayo con ratones (AOAC 1985) y por HPLC (Oshima 1995). Se emplearon las siguientes abreviaturas: Gonyautoxinas 4, 2, 3, y $1=$ GTX4, GTX2, GTX3, GTX1 respectivamente, neosaxitoxina $=$ neoSTX, y saxitoxina $=$ STX. El análisis de TAM por HPLC en todas las muestras de bivalvos se realizó según el método de Wright et al. (1995).

Para el análisis de toxinas en plancton se colectaron muestras de diferentes fracciones de tamaño en la entrada de Bahía A. Brown y Paso R. Guaraní medio. En cada muestra, 201 de agua fueron tamizados sucesivamente por mallas de $150 \mu \mathrm{m}$, $65 \mu \mathrm{m}$ y $20 \mu \mathrm{m}$. El material retenido (fracción 65$20 \mu \mathrm{m})$, fue re suspendido y llevado a un volumen final de $300 \mathrm{~mL}$ con agua de mar filtrada, preservándose una alícuota de $50 \mathrm{~mL}$ de esta suspensión en formol 4\% para el recuento de células fitoplanctónicas. Los $250 \mathrm{~mL}$ restantes se filtraron a través de filtros de fibra de vidrio Whatman GF/F para el análisis de toxinas. 1,5 1 del agua pasada por el tamiz de $20 \mu \mathrm{m}$ se filtró por Whatman GF/F para estudiar las toxinas en esta fracción de plancton (fracción $<20 \mu \mathrm{m}$ ).

\section{Análisis de fitoplancton}

Se tomaron muestras de fitoplancton con red de $20 \mu \mathrm{m}$ de malla mediante barridos horizontales. Una fracción fue mantenida viva para la observación de organismos delicados y formas flageladas, y otra fracción preservada con formaldehído neutralizado al 4\%. También se tomaron alícuotas de $250 \mathrm{ml}$ de agua de superficie que fueron fijadas con solución de Lugol (Utermöhl 1958) para el análisis cuantitativo.
Las muestras de red fueron examinadas con microscopía de campo claro, contraste de fases, contraste diferencial de interferencia (DIC) y epifluorescencia. Las diatomeas fueron tratadas con una solución de $\mathrm{NaClO}$, lavadas con agua destilada (Ferrario et al. 1995) y montadas en Styrax. Se empleó microscopía electrónica de barrido (MEB) para tratar de identificar algunas especies, pero las muestras no pudieron ser observadas satisfactoriamente debido a la muy baja silicificación de los frústulos, que disminuyó marcadamente su resistencia a los tratamientos de limpieza usados rutinariamente (Simonsen 1974; Ferrario et al. 1995).

Para la identificación de dinoflagelados se empleó una tinción de las tecas con Lugol, o mediante Calcofluor según la técnica descripta por Fritz y Trimer (1985). El análisis cuantitativo de las muestras se realizó con un microscopio invertido (Utermöhl 1958).

\section{Aislamiento de especies de microalgas}

Se aislaron microalgas tóxicas presentes en las muestras de fitoplancton destinadas a su cultivo bajo condiciones controladas. Los cultivos fueron mantenidos en medio $\mathrm{L} 1$ a $11{ }^{\circ} \mathrm{C}$ de temperatura, 30 de salinidad y un ciclo de luz/oscuridad de 14/10 h. Los cultivos en fase de crecimiento exponencial fueron utilizados para identificar y cuantificar las diferentes toxinas, la composición de los pigmentos fotosintetizadores y la composición de aminoácidos similares a micosporinas (MAAs) por HPLC.

Para el análisis de MAAs por HPLC, $50 \mathrm{~mL}$ de cada cultivo fueron filtrados por filtros de fibra de vidrio Whatman $\mathrm{GF} / \mathrm{F}$, extraídos con $2 \mathrm{~mL}$ de metanol 100\% siguiendo el método de Carreto et al. (2005). Para el análisis de toxinas y pigmentos por HPLC, alícuotas de $50 \mathrm{ml}$ de cultivo fueron filtrados por filtros de fibra de vidrio Whatman $\mathrm{GF} / \mathrm{F}$, para luego continuar con la metodología descripta para el análisis de fitoplancton. 


\section{RESULTADOS}

\section{Características ambientales}

Se registraron 14 perfiles de CTD en Bahía A. Brown y su sistema de drenaje para conocer la distribución vertical y horizontal de la temperatura, salinidad y densidad. La profundidad del fondo varió entre una máxima de $24 \mathrm{~m}$ en la entrada de Bahía A. Brown (estaciones 6 y 7), y una mínima de 1,5 m en el Paso R. Guaraní (estación 14).

La distribución superficial de temperatura mostró un incremento gradual hacia el interior de la bahía (Figura $2 \mathrm{~A})$. La menor temperatura $\left(7,19^{\circ} \mathrm{C}\right)$ se registró en la entrada, en el sector adyacente a Isla Gable, relacionada al ingreso de agua proveniente del flujo principal del canal. En el sector interior de la bahía se registró una temperatura de 7,68 ${ }^{\circ} \mathrm{C}$ en las proximidades de la Bahía A. Brown. En el Paso R. Guaraní se registraron temperaturas mayores de $8{ }^{\circ} \mathrm{C}$, formando en su entrada un gradiente térmico con el agua de la bahía (este de la Isla Upú). Hacia el interior del paso, la temperatura alcanzó un máximo de $8,9{ }^{\circ} \mathrm{C}$, en correspondencia con la mínima profundidad del área $(1,5 \mathrm{~m})$, disminuyendo nuevamente hacia el Este por influencia del agua del canal.

La distribución superficial de salinidad mostró un gradiente superficial muy marcado en la zona externa de la bahía, donde se registró la menor salinidad del área $(29,74)$ en el sector costero cercano a Punta Almanza, debida a la desembocadura del Río Almanza situada a corta distancia de la estación 5 (Figura 2 B). Inversamente, sobre el margen opuesto (Isla Gable) se registró la mayor salinidad del área $(30,67)$ relacionada al agua externa del canal. A partir de este sector externo, la salinidad disminuyó gradualmente hacia el interior de la bahía $(30,31)$ y hacia el Paso R. Guaraní, donde se registraron salinidades alrededor de 30,2.
La distribución vertical de los parámetros ambientales mostró que la capa superficial de baja salinidad, registrada en la entrada de la bahía, ocupó los primeros 1,5 $\mathrm{m}$ de profundidad en la estación 5 (Figura 3). La baja salinidad del agua $(29,75)$ determinó la menor densidad (Sigma-T) de esta capa $\left(\sigma_{t}=23,2 \mathrm{~kg} \mathrm{~m}^{-3}\right)$, formando una picnoclina con el agua inferior con una densidad

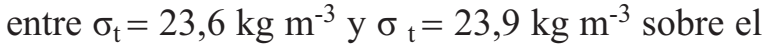
fondo. Esta capa superficial de menor densidad se manifestó débilmente en el registro térmico, el cual mostró una leve termoclina $\left(0,1{ }^{\circ} \mathrm{C} \mathrm{m}^{-1}\right)$. Debajo de esta, se observó una disminución gradual de la temperatura con la profundidad (entre $7,4{ }^{\circ} \mathrm{C}$ y $7,0^{\circ} \mathrm{C}$ ) hasta los $16 \mathrm{~m}$. A partir de esta profundidad, se observó una capa de mayor densidad $\left(\sigma_{\mathrm{t}}=24,06 \mathrm{~kg} \mathrm{~m}^{-3}\right)$ donde la temperatura y la salinidad se mantuvieron casi constantes hasta el fondo (temperatura: $6,8{ }^{\circ} \mathrm{C}$, salinidad: 30,71 ). Hacia la zona media de la entrada (estación 6) la señal de baja salinidad superficial se vio debilitada $(30,24)$, incrementándose la densidad de la capa superficial $\left(\sigma_{\mathrm{t}}=23,61 \mathrm{~kg} \mathrm{~m}^{-3}\right)$, y atenuada completamente $(30,65)$ en el margen opuesto (Isla Gable) (estación 7). En esta estación, se diferenció una capa superficial hasta los 9,5 m de profundidad, definida principalmente por la temperatura. La capa de mezcla presentó una salinidad de 30,66 y una temperatura de $7,2^{\circ} \mathrm{C}$, separada por una leve termoclina $\left(0,1^{\circ} \mathrm{C} \mathrm{m}^{-1}\right)$ de la capa de fondo (salinidad: 30,75 , temperatura: $6,8{ }^{\circ} \mathrm{C}$ ) a partir de los $12 \mathrm{~m}$ de profundidad.

En la zona central de la bahía, la señal de baja salinidad superficial registrada en la estación 5 se encontró también atenuada en la estación más cercana a la costa de Puerto Almanza (estación 8), con valores de 30,42 hasta $3,5 \mathrm{~m}$ de profundidad. No obstante, el perfil de salinidad en esta estación mostró dos haloclinas La primera se localizó entre $\operatorname{los} 3,5$ y $7 \mathrm{~m}$ de profundidad, asociada a la capa superficial de baja salinidad proveniente de la desembocadura del Río Almanza (Figura 4), y la segunda se registró a los $17 \mathrm{~m}$, separando el agua de profundidad intermedia de una capa de 


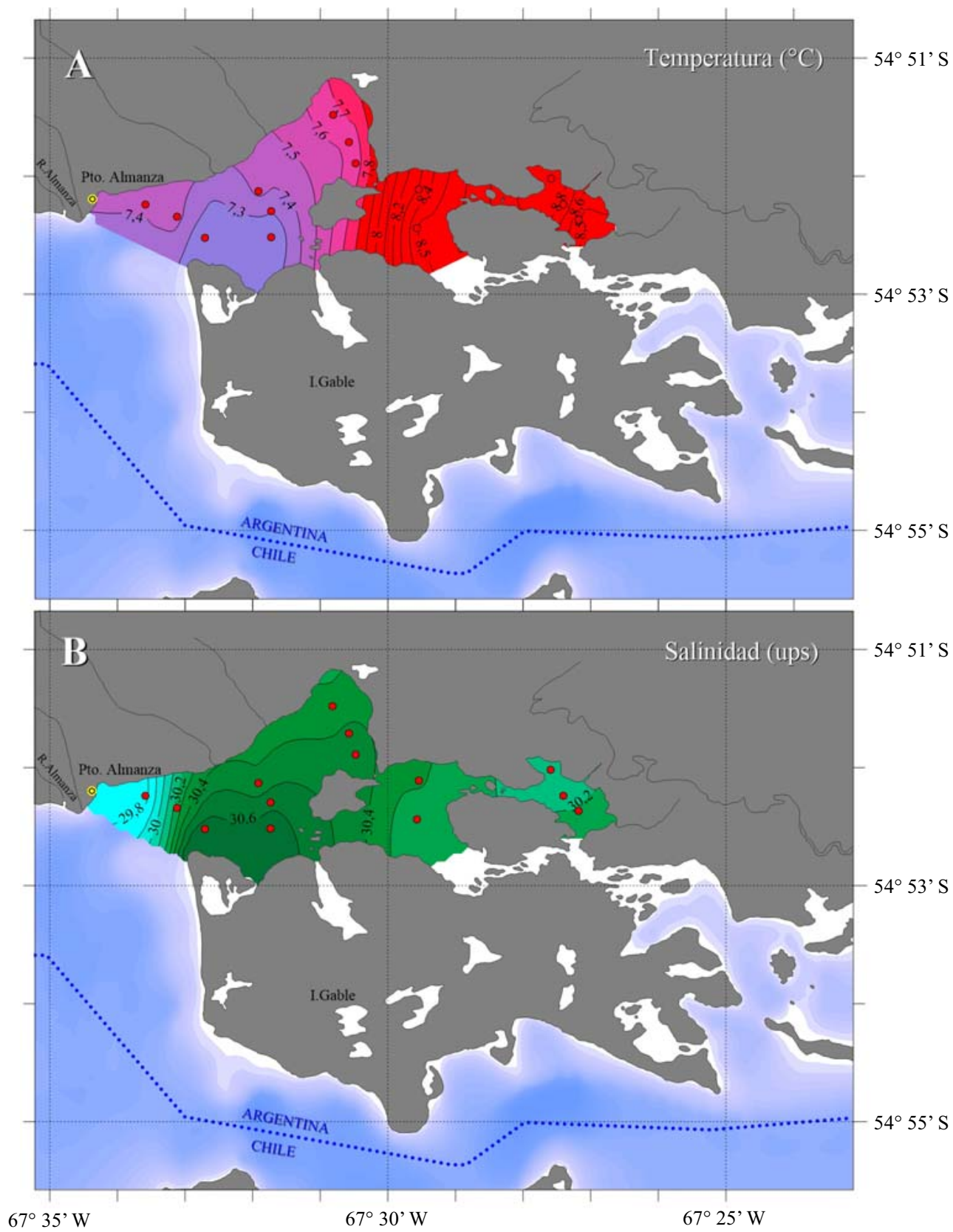

Figura 2. Distribución superficial de temperatura $\left({ }^{\circ} \mathrm{C}\right)(\mathrm{A})$ y salinidad (B) en el área de Bahía A. Brown y su sistema de drenaje. Figure 2. Surface distribution of temperature $\left({ }^{\circ} \mathrm{C}\right)(\mathrm{A})$ and salinity $(B)$ in the area of $A$. Brown Bay and its drainage system.

fondo de mayor salinidad (> 30,7), la cual se encontró definida en el registro térmico por una leve termoclina a $17 \mathrm{~m}$ de profundidad (Figura 4) y 3,5 m sobre el fondo.
En la estación central (estación 9), la señal de baja salinidad superficial se manifestó débilmente hasta $0,5 \mathrm{~m}$. La capa de fondo, de mayor densi$\mathrm{dad}$, fue registrada a partir de $18 \mathrm{~m}$, con un espe- 
Estación 5

Temperatura $\left({ }^{\circ} \mathrm{C}\right)$

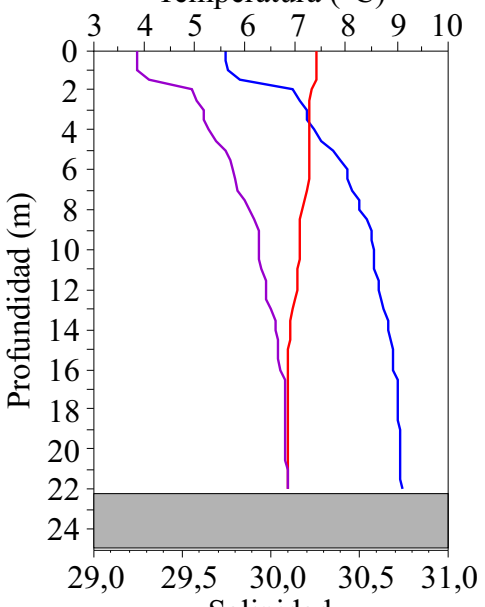

Salinidad

$23,0 \quad 23,4$ 23,8 24,2 24,6 25,0

Densidad $\left(\mathrm{kg} \mathrm{m}^{-3}\right)$
Estación 6

Temperatura $\left({ }^{\circ} \mathrm{C}\right)$

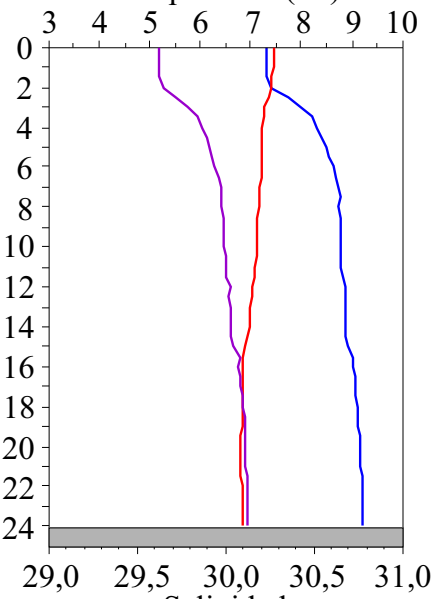

Salinidad

$23,023,423,8 \quad 24,2 \quad 24,6$

Densidad $\left(\mathrm{kg} \mathrm{m}^{-3}\right)$
Estación 7

Temperatura $\left({ }^{\circ} \mathrm{C}\right)$

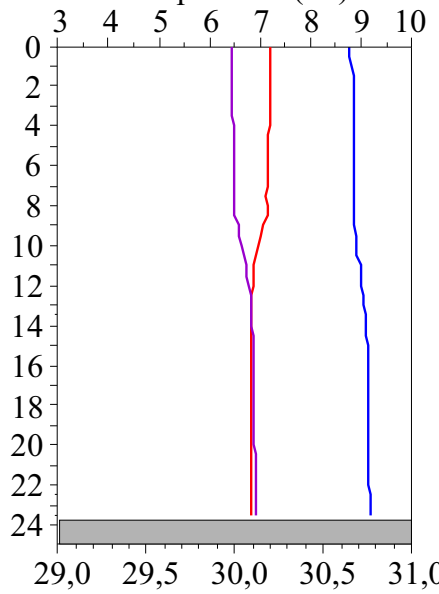

Salinidad

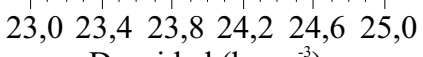

Densidad $\left(\mathrm{kg} \mathrm{m}^{-3}\right)$

Figura 3. Distribución vertical de temperatura, salinidad y densidad $\sigma_{\mathrm{t}}$ en las estaciones 5, 6 y 7 ubicadas en la entrada de Bahía A. Brown.

Figure 3. Vertical distribution of temperature, salinity and density $\sigma_{t}$ in stations 5, 6 and 7 located at the entrance of A. Brown Bay.

Estación 8

Temperatura $\left({ }^{\circ} \mathrm{C}\right)$

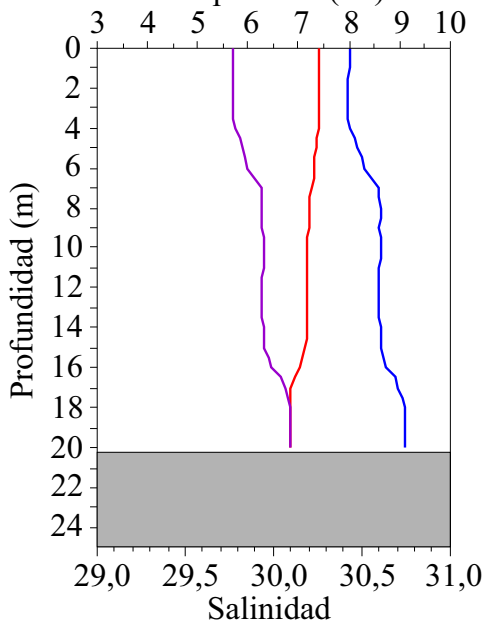

$23,0 \quad 23,4 \quad 23,8 \quad 24,2 \quad 24,6 \quad 25,0$ Densidad $\left(\mathrm{kg} \mathrm{m}^{-3}\right)$
Estación 9

Temperatura $\left({ }^{\circ} \mathrm{C}\right)$

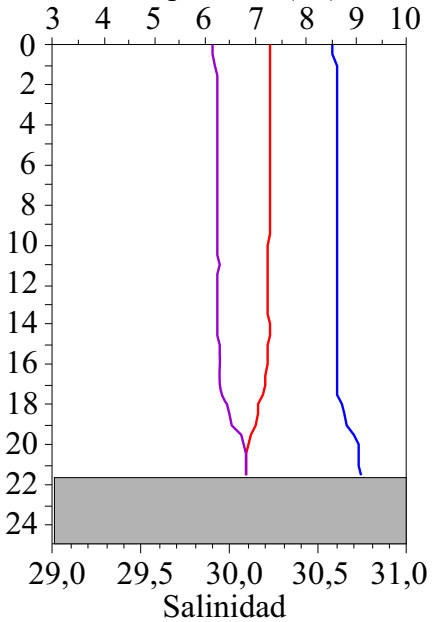

$23,023,4 \quad 23,8 \quad 24,2 \quad 24,6 \quad 25,0$

Densidad $\left(\mathrm{kg} \mathrm{m}^{-3}\right)$
Estación 10

Temperatura $\left({ }^{\circ} \mathrm{C}\right)$

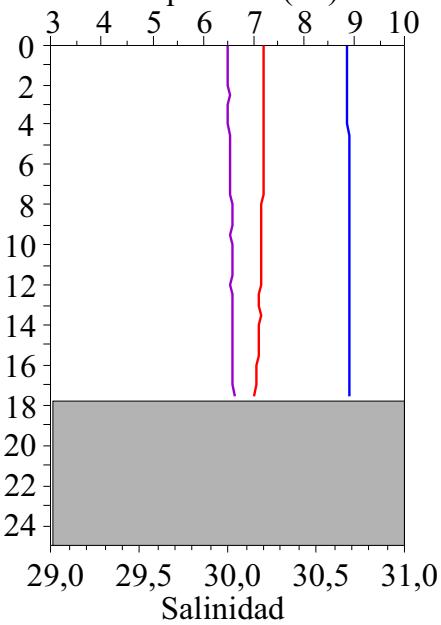

$23,023,423,824,2 \quad 24,625,0$

Densidad $\left(\mathrm{kg} \mathrm{m}^{-3}\right)$

Figura 4. Distribución vertical de temperatura, salinidad y densidad $\sigma_{\mathrm{t}}$ en las estaciones 8,9 y 10 realizadas en el sector medio de Bahía A. Brown.

Figure 4. Vertical distribution of temperature, salinity and density $\sigma_{t}$ at stations 8,9 and 10 carried out in the middle sector of A. Brown Bay. 
sor de $2 \mathrm{~m}$ sobre el fondo. En la estación 10, próxima a la costa de la Isla Gable, los parámetros ambientales mostraron una completa homogeneidad vertical (salinidad: 30,68 , temperatura: $7,1^{\circ} \mathrm{C}$ ) (Figura 4).

En el sector interior de la bahía (estaciones 11, 12 y 13) la circulación más restringida posibilitó el desarrollo de una capa superficial más definida por la menor salinidad $(30,31)$ y mayor temperatura $\left(7,6{ }^{\circ} \mathrm{C}\right)$, limitada por una termoclina a $9 \mathrm{~m}$ de profundidad $\left(0,3{ }^{\circ} \mathrm{C} \mathrm{m}^{-1}\right)$ (Figura 5). En la estación 11, próxima a Bahía A. Brown, la distribución vertical de salinidad mostró una leve disminución en el primer metro superficial, probablemente debida al aporte de agua de deshielo producido por los arroyos y chorrillos que desembocan en la costa.

Los perfiles realizados en el Paso R. Guaraní mostraron una completa mezcla vertical (estaciones 14, 15, 16 y 18) (Figuras 6 y 7) debida a la baja profundidad; excepto el correspondiente a la estación 17 (Figura 7) próxima a la Punta Lápiz, donde se observó una leve disminución de la salinidad superficial similar a la observada en la estación 11. Las temperaturas registradas en todo este sector fueron mayores de $8,0^{\circ} \mathrm{C}$. Las mediciones realizadas en el Paso R. Guaraní medio se caracterizaron además por la menor densidad del agua, determinada principalmente por la mayor temperatura, con un máximo de $8,9^{\circ} \mathrm{C}$ en la estación de más baja profundidad (estación 14) (Figura 6).

\section{Nutrientes inorgánicos}

En forma general, se observó una alta concentración de silicato en todas las muestras analizadas (> 10,7 $\mu \mathrm{M}$ ), producida por el aporte de aguas de deshielo continental a través de los ríos y chorrillos que desembocan en la costa del canal. El anión fosfato presentó bajas concentraciones $(<0,2 \mu \mathrm{M})$ y escasa variabilidad entre estaciones y en el tiempo. Esta escasa variabilidad es también

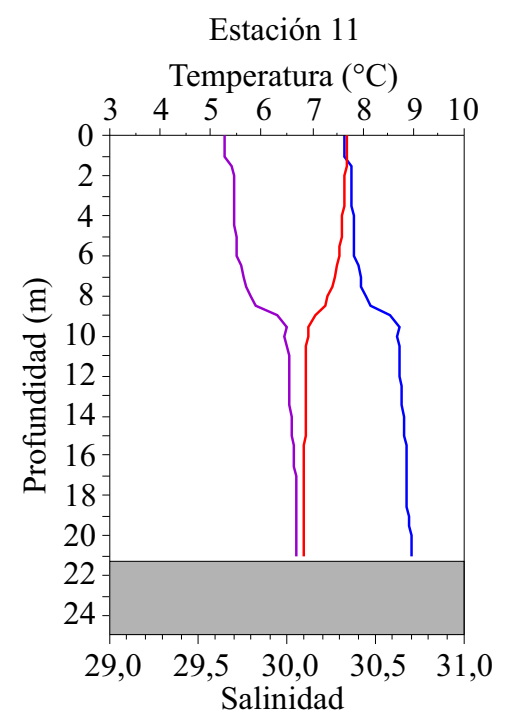

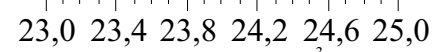
Densidad $\left(\mathrm{kg} \mathrm{m}^{-3}\right)$

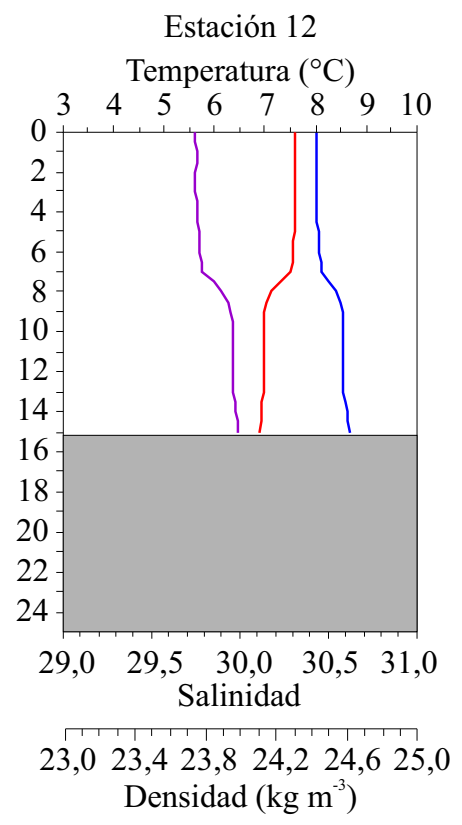

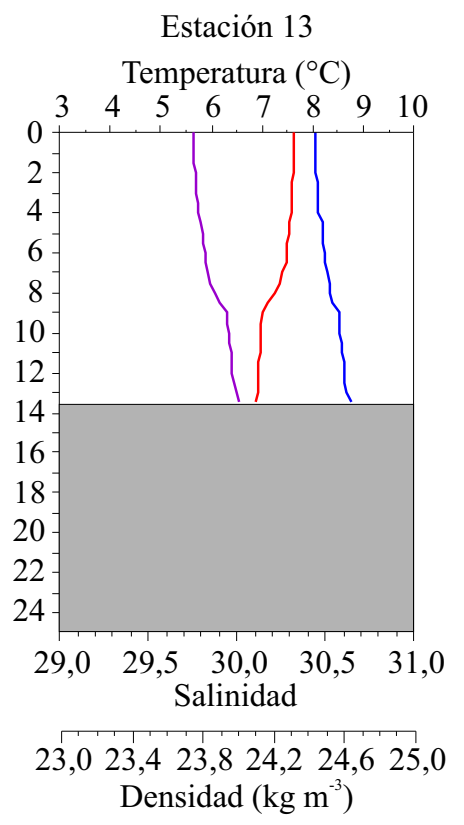

Figura 5. Distribución vertical de temperatura, salinidad y densidad $\sigma_{\mathrm{t}}$ en las estaciones 11, 12 y 13 realizadas en el sector interior de Bahía A. Brown.

Figure 5. Vertical distribution of temperature, salinity and density $\sigma_{t}$ at stations 11,12 and 13 carried out in the inner sector of A. Brown Bay. 
Estación 14

Temperatura $\left({ }^{\circ} \mathrm{C}\right)$

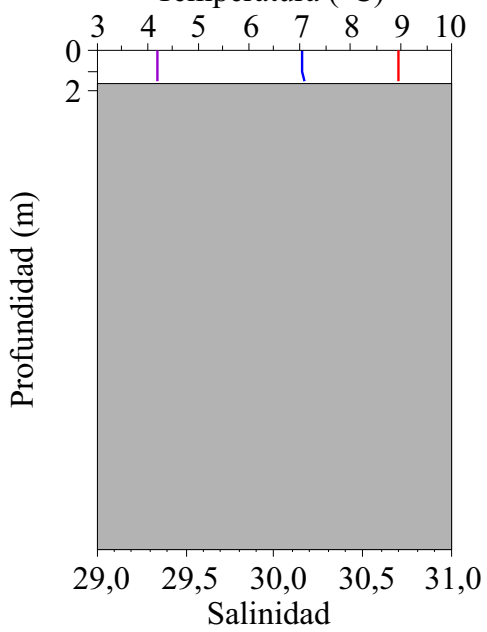

$23,023,423,824,2$ 24,6 25,0 Densidad $\left(\mathrm{kg} \mathrm{m}^{-3}\right)$
Estación 15

Temperatura $\left({ }^{\circ} \mathrm{C}\right)$

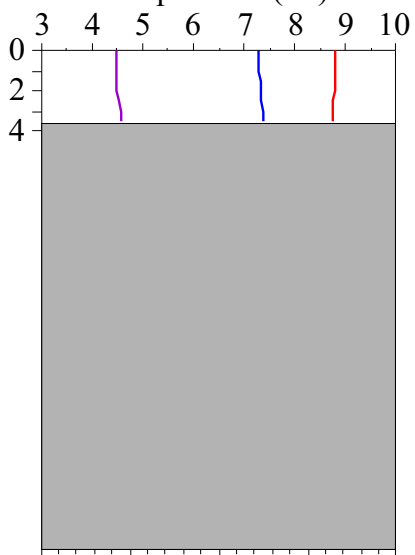

$29,0 \quad 29,5 \quad 30,0 \quad 30,5 \quad 31,0$

Salinidad

$23,023,4 \quad 23,8 \quad 24,2 \quad 24,6 \quad 25,0$

Densidad $\left(\mathrm{kg} \mathrm{m}^{-3}\right)$
Estación 16
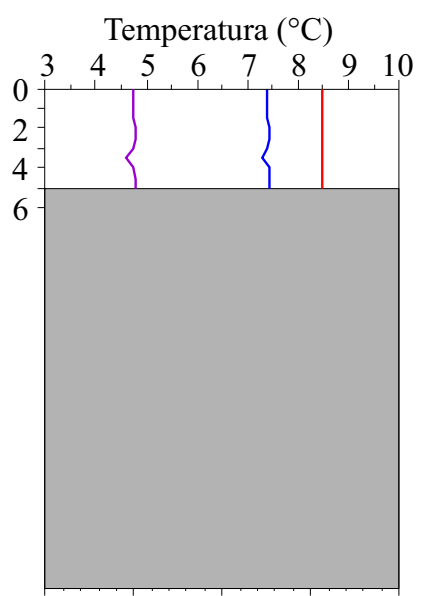

$29,0 \quad 29,5 \quad 30,0 \quad 30,5 \quad 31,0$

Salinidad

$23,023,423,824,2 \quad 24,6 \quad 25,0$

Densidad $\left(\mathrm{kg} \mathrm{m}^{-3}\right)$

Figura 6. Distribución vertical de temperatura, salinidad y densidad $\sigma_{t}$ en las estaciones 14, 15 y 16 realizadas en Paso R. Guaraní medio.

Figure 6. Vertical distribution of temperature, salinity and density $\sigma_{t}$ at stations 14,15 and 16 carried out in the middle of R. Guarani Strait.

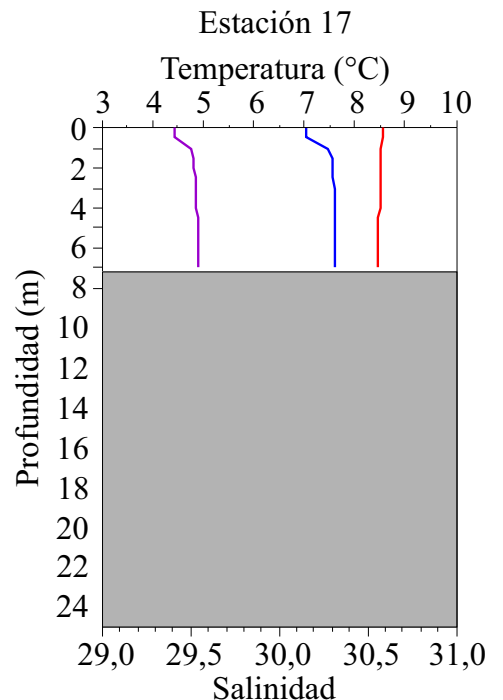

$23,0 \quad 23,4 \quad 23,8 \quad 24,2 \quad 24,6 \quad 25,0$

Densidad $\left(\mathrm{kg} \mathrm{m}^{-3}\right)$
Estación 18

Temperatura $\left({ }^{\circ} \mathrm{C}\right)$

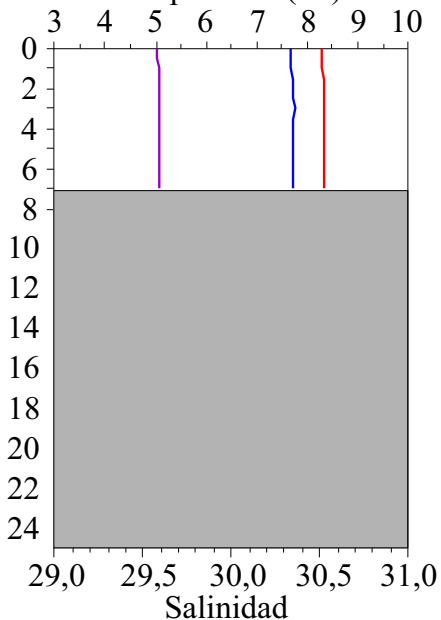

$23,023,4 \quad 23,8 \quad 24,2 \quad 24,6 \quad 25,0$

Densidad $\left(\mathrm{kg} \mathrm{m}^{-3}\right)$

Figura 7. Distribución vertical de temperatura, salinidad y densidad $\sigma_{t}$ en las estaciones 17 y 18 realizadas en Paso R. Guaraní entre la Isla Upú y la Isla Warú.

Figure 7. Vertical distribution of temperature, salinity and density $\sigma_{t}$ at stations 17 and 18 carried out in R. Guarani Strait between Upú Island and Warú Island. 
común para el anión nitrito. La Bahía A. Brown presentó los valores máximos y mínimos de nitrato registrados $(11,21 \mu \mathrm{M}$ y $0,56 \mu \mathrm{M})$ mostrando una brusca disminución de los mismos en el tiempo, propiedad compartida con el Paso R. Guaraní (Tabla 1). Este marcado descenso en la concentración del nitrato probablemente tenga su explicación en el consumo de este macronutriente ocasionado por el crecimiento del fitoplancton del área durante este período.

\section{Análisis de la información climatológica}

Los registros atmosféricos obtenidos por la estación meteorológica 879380 (SAWH) del aeropuerto de la ciudad de Ushuaia también mostraron que, tanto la temperatura media como la máxima media y la mínima media durante los meses de primavera de 2012 a 2014, estuvieron por debajo de las respectivas medias históricas. y el valor promedio de la velocidad del viento en este período fue superior al valor medio histórico (Figura 8).

\section{Análisis de fitoplancton}

Se identificó un total de 64 especies de fitoplancton, pertenecientes a los grupos de diatomeas, dinoflagelados, silicoflagelados, Cryptophytas, Prasinophytas y Cyanoprocariotas (Tabla 2).

Los recuentos celulares mostraron una domi- nancia casi completa de diatomeas en las muestras obtenidas en el área de Bahía A. Brown el día 10 de diciembre, con una abundancia relativa de $98,8 \%$. El resto de los grupos observados presentaron una muy baja abundancia relativa, con niveles menores de $1 \%$, excepto en la muestra colectada en el Paso R. Guaraní medio, donde las Prasinophytas mostraron una abundancia relativa de $1,8 \%$.

En las muestras colectadas en el área de Bahía A. Brown una semana después (17 de diciembre), las diatomeas mostraron también una dominancia muy marcada dentro de la comunidad fitoplanctónica, con una abundancia relativa alrededor del 98\% en la entrada de la bahía y en el Paso R. Guaraní medio, que disminuyó en el interior de la bahía $(84,1 \%)$ debido a una población de una Cyanoprocariota solitaria, Synechocystis cf. sali$n a$, que alcanzó una abundancia relativa de $15,2 \%$. A pesar de su abundancia relativa en términos de biomasa, su abundancia fue poco significativa debido al reducido tamaño de las células de Synechocystis (2,5 $\mu \mathrm{m}$ de diámetro). En la muestra tomada en la estación contigua (Punta Lápiz) la comunidad fitoplanctónica presentó una estructura similar, con una abundancia relativa de diatomeas de $86,1 \%$ y de Synechocystis de $12,7 \%$. La concentración celular de Synechocystis en el plancton disminuyó marcadamente en la muestra tomada en el Paso R. Guaraní medio (abundancia relativa $0,8 \%$ ), donde las diatomeas

Tabla 1. Concentración de nutrientes inorgánicos disueltos en la superficie de las estaciones seleccionadas.

Table 1. Concentration of inorganic nutrients dissolved on the surface of the selected stations.

\begin{tabular}{llccccc}
\hline Estación & Lugar & Fecha & $\begin{array}{c}\text { Nitrato } \\
(\mu \mathrm{M})\end{array}$ & $\begin{array}{c}\text { Nitrito } \\
(\mu \mathrm{M})\end{array}$ & $\begin{array}{c}\text { Fosfato } \\
(\mu \mathrm{M})\end{array}$ & $\begin{array}{c}\text { Silicato } \\
(\mu \mathrm{M})\end{array}$ \\
\hline 6 & Bahía A. Brown (entrada) & $10-12-2013$ & 11,21 & 1,99 & 0,20 & 32,15 \\
22 & Bahía A. Brown (entrada) & $17-12-2013$ & 0,56 & 1,36 & 0,10 & 10,70 \\
14 & Paso R. Guaraní medio & $10-12-3013$ & 8,76 & 1,01 & 0,19 & 23,39 \\
25 & Paso R. Guaraní medio & $17-12-2013$ & 1,74 & 1,58 & 0,12 & 13,78 \\
\hline
\end{tabular}




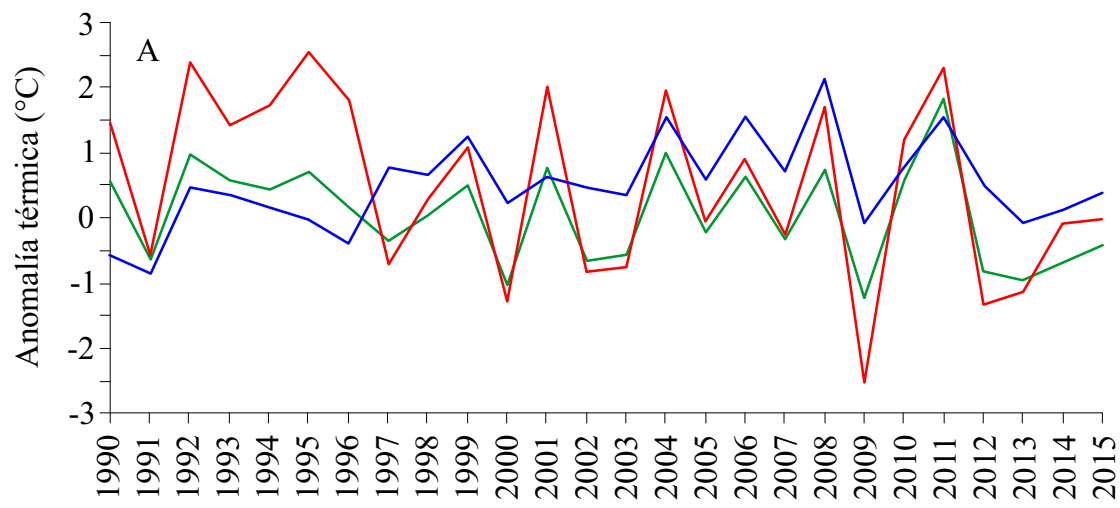

Año

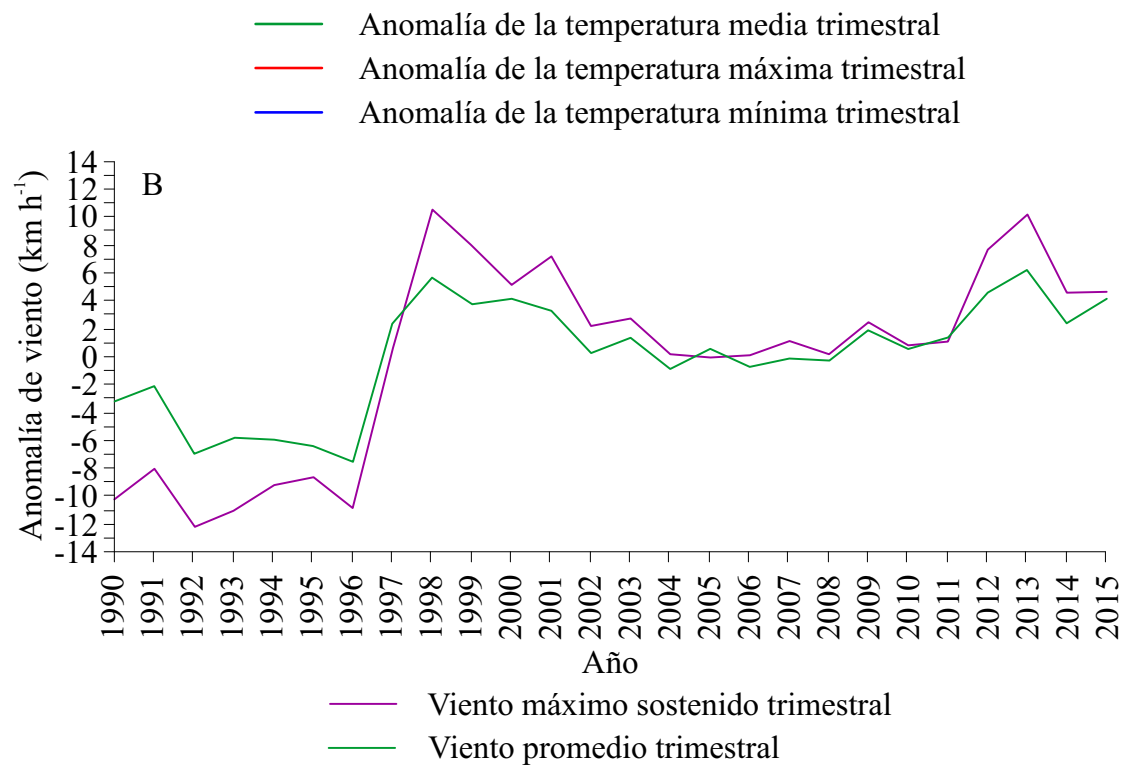

Figura 8. Anomalías de temperatura (A) y viento (B). Registros históricos reportados por la estación meteorológica aeroportuaria de Ushuaia (879380 SAWH). Septiembre-noviembre 1990-2015.

Figure 8. Temperature (A) and wind (B) anomalies. Historical records reported by the Ushuaia airport meteorological station (879380 SAWH). September-November 1990-2015.

dominaron completamente la comunidad $(98,1 \%)$ (Tabla 3).

Los recuentos celulares mostraron que la alta dominancia de diatomeas en las muestras tomadas el día 10 de diciembre en el área de Bahía $\mathrm{A}$. Brown fue principalmente debida a cuatro especies del Género Chaetoceros: $C$. socialis, $C$. decipiens, $C$. lorenzianus y $C$. similis. En la entrada de la bahía se registró una densidad celular entre $1,2 \times 10^{6}$ cél.

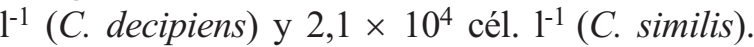

Tanto en el interior de la bahía como en las muestras tomadas en Punta Lápiz y en el Paso R. Guaraní medio, la densidad celular presentó un rango

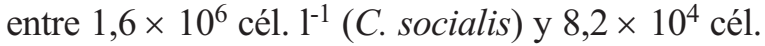
$1^{-1}$ (C. similis). El resto de las especies de diatomeas presentes en las muestras mostraron densidades menores que $1 \times 10^{4}$ cél. $1^{-1}$, excepto Leptocylindrus mínimum en la entrada de la Bahía A. Brown

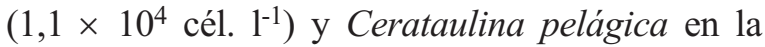

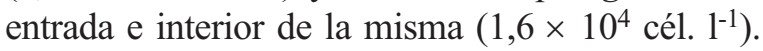




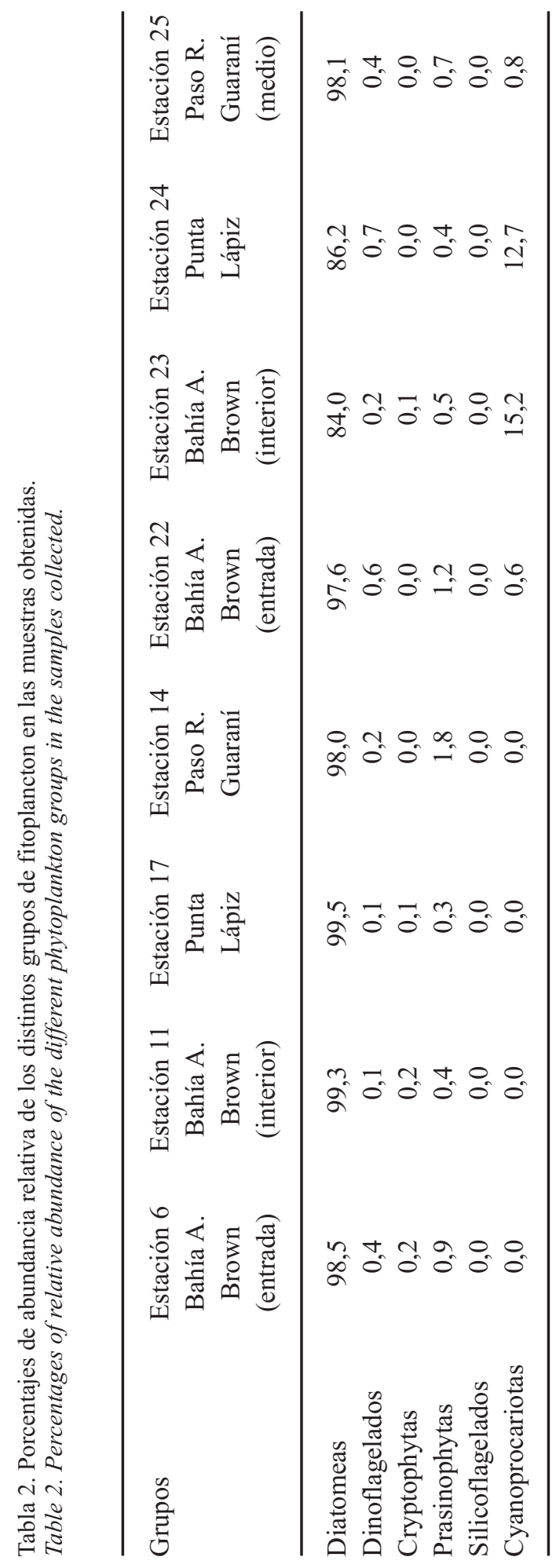

Otros grupos fitoplanctónicos no mostraron una concentración celular significativa, salvo las Prasinophytas en la entrada e interior de Bahía A. Brown, principalmente representadas por Pyra-

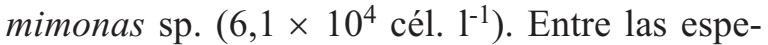
cies tóxicas o potencialmente tóxicas de fitoplancton solo se observó una muy baja densidad de los dinoflagelados A. ostenfeldii y Prorocentrum minimum en la entrada e interior de la

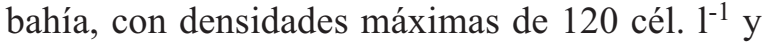
7.147 cél. $1^{-1}$ respectivamente; y $P$. lima en la entrada de la bahía y el Paso R. Guaraní medio (1.021 cél. $\left.^{-1}\right)$. También se registraron densidades significativas de cuatro especies de diatomeas del género Pseudo-nitzschia, potencialmente productoras de toxina amnésica de moluscos (TAM), con una densidad máxima de $1,0 \times 10^{5}$ cél. $1^{-1}$ en el interior de la bahía.

En las muestras tomadas el día 17 de diciembre en Bahía A. Brown, también se observó una marcada dominancia de las especies de Chaetoceros mencionadas con una disminución en sus densida-

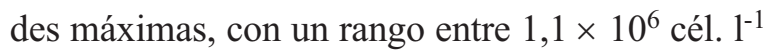

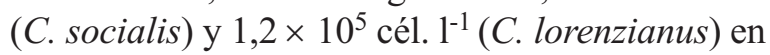
Punta Lápiz. En el Paso R. Guaraní medio, $C$. decipiens y $C$. similis presentaron una densidad máxima de $3,1 \times 10^{4}$ cél. $1^{-1}$. También se observó un aumento significativo en la densidad de las diatomeas Striatella unipunctata $\left(2,3 \times 10^{5}\right.$ cél. $\left.1^{-1}\right)$, Leptocylindus danicus $\left(2,3 \times 10^{5}\right.$ cél. $\left.1^{-1}\right)$ y $L$. minimus $\left(2,3 \times 10^{5}\right.$ cél. $\left.1^{-1}\right)$ respecto al muestreo previo. La Prasinophyta Pyramimonas sp. mostró una disminución de densidad, principalmente en la entrada de la bahía y en el Paso R. Guaraní medio. Considerando las especies de fitoplancton tóxicas o potencialmente tóxicas, se observó la presencia de $A$. ostenfeldii en toda el área, aunque con muy baja densidad. La máxima densidad (320 cél. $\left.1^{-1}\right)$ se registró en el interior de la bahía, disminuyendo hacia la entrada (80 cél. $\left.\mathrm{l}^{-1}\right)$ y hacia el Paso R. Guaraní medio (200 cél. 1 $^{-1}$ ). P. lima solo fue registrado en el Paso R. Guaraní medio (40 cél. $\mathrm{1}^{-1}$ ) y $P$. minimum presentó mayores densidades en el interior de la bahía y Punta Lápiz (4.084 
Tabla 3. Especies identificadas en las muestras de fitoplancton.

Table 3. Species identified in the phytoplankton samples.

\begin{tabular}{|c|c|}
\hline Diatomeas & Dinoflagelados \\
\hline Amphora sp. & Alexandrium ostenfeldii \\
\hline Chaetoceros decipiens & Anphidinium carterae \\
\hline Chaetoceros lorenzianus & Gymnodinium spp. \\
\hline Chaetoceros similis & Gyrodinium fusus \\
\hline Chaetoceros diadema & Gyrodinium sp. \\
\hline Chaetoceros socialis & Oxytoxum sp. \\
\hline Chaetoceros cf. convolutus & Prorocentrum lima \\
\hline Corethron kriophyllum & Prorocentrum compressum \\
\hline Corethron histrix & Prorocentrum minimum \\
\hline Coscinodiscus radiatus & Protoperidinium cuspidatum \\
\hline Coscinodiscus sp. & Protoperidinium metananum \\
\hline Ceratoneis closterium & Protoperidinium nudum \\
\hline Cerataulina pelagica & Protoperidinium simulum \\
\hline Fragilaria sp. & Protoperidinium sp. \\
\hline Guinardia delicatula & Scrippsiella patagonica \\
\hline Haslea sp. & Cyanoprocariotas \\
\hline Leptocylindrus danicus & Synechocystis cf. salina \\
\hline Leptocylindrus minimus & Cryptophytas \\
\hline Lycmophora sp. & Cryptomnadales \\
\hline Navicula sp. & Silicoflagelados \\
\hline Thalassiothrix sp. & Dictiocha speculum \\
\hline Pleurosigma normanii & Prasinophytas \\
\hline Pseudo-nitzschia spp. & Tetraselmis sp. \\
\hline Rhizosolenia setigera & Pyramimonas sp. \\
\hline Skeletonema costatum & Ciliophora \\
\hline Stephanopixis turris & Myrionecta rubra \\
\hline \multicolumn{2}{|l|}{ Sriatella unipunctata } \\
\hline Thalassiosira sp. & \\
\hline Thalassionema nitzschioid & \\
\hline
\end{tabular}

cél. $\left.1^{-1}\right)$. Las especies del Género Pseudo-nitzschia presentaron densidades similares a las registradas en las muestras tomadas el día 10 de diciembre, con una leve disminución en el Paso R. Guaraní medio $\left(9,4 \times 10^{3}\right.$ cél. $\left.1^{-1}\right)$.

Otras diferencias significativas respecto al muestreo anterior fueron la presencia de Synechocystis $\mathrm{cf}$. salina, cuyas mayores densidades se ubicaron en el interior de la bahía y Punta Lápiz

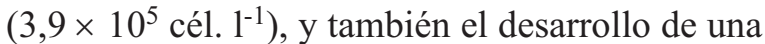
población del ciliado Myrionecta rubra en toda el área de Bahía A. Brown con una densidad máxima de $2,7 \times 10^{4}$ cél. $1^{-1}$ en el interior de la bahía. Si bien $M$. rubra no pertenece a la comunidad fitoplanctónica, fue considerado en los recuentos celulares por ser un ciliado fotosintético con plás- 
tidos endosimbióticos, directamente relacionado a varias especies tóxicas del genero Dinophysis que constituyen una presa indispensable para su crecimiento (Nagai et al. 2008).

\section{Análisis de pigmentos}

Se identificaron 15 pigmentos diferentes en las cuatro muestras analizadas (Tabla 4). La mayor concentración de clorofila $a\left(5,298 \mu \mathrm{g} \mathrm{l}^{-1}\right)$ se halló en la muestra tomada el 17 de diciembre en la entrada de Bahía A. Brown (estación 22), donde se encuentra la balsa de cultivo de mejillones (Tabla 4). Esta biomasa fitoplanctónica se corresponde con la mayor concentración de diatomeas, por lo que también coincide con la mayor concentración de fucoxantina $\left(2,11 \mu \mathrm{g} \mathrm{l}^{-1}\right)$. Otros pigmentos marcadores de grupos fitoplanctónicos fueron hallados en menor proporción. Peridinina, carotenoide marcador de dinoflagelados, solo fue detectado en Bahía A. Brown el 17 de diciembre, mientras que 19'-hexanoiloxifucoxantina (Hexfuco), marcador de Haptophytas, fue detectado únicamente en Paso R. Guaraní medio el 10 de diciembre. Zeaxantina, presente principalmente en Cyanoprocariotas, solo fue hallado en el Paso R. Guaraní medio el 10 de diciembre, aunque este pigmento es fuertemente variable dependiendo de las condiciones de luz.

\section{Análisis de toxinas}

Dado que no se registraron concentraciones importantes de microalgas nocivas en ninguna de las estaciones examinadas, el análisis de toxinas por HPLC en las diferentes fracciones de fitoplancton se descartó. Para evaluar la evolución de la toxicidad en el Canal Beagle, se realizó el aná-

Tabla 4. Concentración de pigmentos $\left(\mu \mathrm{g}^{-1}\right)$ en Bahía A. Brown y Paso R. Guaraní.

Table 4. Pigment concentration $\left(\mu \mathrm{g}^{-1}\right)$ in A. Brown Bay and R. Guarani Strait.

\begin{tabular}{lcccc}
\hline Pigmento & $\begin{array}{c}\text { Estación } 6 \\
\text { Bahía A. Brown } \\
\text { 10 de diciembre }\end{array}$ & $\begin{array}{c}\text { Estación 14 } \\
\text { Paso R. Guaraní } \\
\text { 10 de diciembre }\end{array}$ & $\begin{array}{c}\text { Estación 22 } \\
\text { Bahía A. Brown } \\
17 \text { de diciembre }\end{array}$ & $\begin{array}{c}\text { Estación 25 } \\
\text { Paso R. Guaraní } \\
\text { 17 de diciembre }\end{array}$ \\
\hline Clorofila $c_{3}$ & 0,012 & & 0,024 & 0,010 \\
Clorofilida & 0,025 & 0,072 & 0,119 & 0,073 \\
MGDVP & 0,034 & 0,019 & 0,034 & 0,012 \\
Clorofila $c_{2}$ & 0,254 & 0,207 & 0,711 & 0,290 \\
Clorofila $c_{1}$ & 0,078 & 0,262 & 0,310 & 0,122 \\
Peridinina & 0,765 & 0,677 & 0,072 & 0,890 \\
Fucoxantina & 0,091 & 0,182 & 2,110 & 0,151 \\
Hex-fuco & 0,156 & 0,260 & 0,011 \\
Diadinoxantina & 0,035 & 0,024 & 0,022 & 0,026 \\
Alloxantina & & & 0,033 & \\
Diatoxantina & & 0,014 & 0,030 & 2,042 \\
Zeaxantina & 0,048 & 0,128 & 5,298 & 0,032 \\
Clorofila $b$ & 2,062 & 1,857 & 0,065 & \\
Clorofila $a$ & 0,027 & 0,047 & & \\
$\beta \beta$-caroteno & & & & \\
\hline
\end{tabular}


lisis de la presencia de TPM y TAM en una serie de muestras de cholgas y mejillones colectadas en meses previos y analizadas por bioensayo en el Laboratorio Ambiental de la Secretaria de Ambiente, Desarrollo Sostenible y Cambio Climático de la Provincia de Tierra del Fuego, Antártida e Islas del Atlántico Sur. Se analizaron un total de 21 muestras para la detección de TPM, de las cuales siete dieron resultados negativos por bioensayo. No obstante, en todas fue posible detectar toxinas en diferente concentración utilizando el método de HPLC de mayor sensibilidad. Se realizaron los cálculos de toxicidad a partir de las concentraciones de cada una de las toxinas halladas en las muestras y los factores individuales determinados por Oshima (1995). El coeficiente de correlación entre los resultados calculados por estas dos formas de análisis (expresados como $\mu \mathrm{g}$ de STX eq. $\mathrm{kg}^{-1}$ de tejido de moluscos) fue bajo $(\mathrm{r}=0,466)$, lo que demuestra la diferencia de sensibilidad entre ambos métodos a bajas concentraciones de toxinas (Figura 9).

En los resultados de los análisis por HPLC se observó en general que para la misma fecha en muestras provenientes de la balsa ubicada en la entrada de la Bahía A. Brown (Figura 10), las cholgas presentan una mayor concentración de TPM que los mejillones.

La toxicidad y la cantidad de toxinas totales en las muestras de cholgas como de mejillones descendieron durante el período analizado. Sin embargo fue posible observar un aumento de toxicidad y concentración total de toxinas tanto en mejillones como en cholgas en las muestras colectadas en la misma balsa el 2 de septiembre y el 2 de diciembre de 2013. La muestra de cholgas correspondiente al 2 de septiembre alcanzó la máxima concentración de toxinas totales halladas (2.018 $\mu$ g STX eq. $\mathrm{kg}^{-1}$ ) (Figura 10).

El perfil tóxico de las muestras, tanto de mejillones como de cholgas, fue variable y detectó gonyautoxinas GTX1, GTX2, GTX3 y GTX4 como las principales toxinas presentes (Figura 11). Dada la rápida epimerización que ocurre en este grupo de toxinas, se expresaron los porcentajes agrupándolos en pares epiméricos (GTX2/3 y GTX1/4). Otras toxinas detectadas fueron las muy tóxicas neoSTX y STX. Estas últimas solo estaban presentes en las muestras de mejillones colectadas el 2 y 17 de diciembre en la balsa, lo

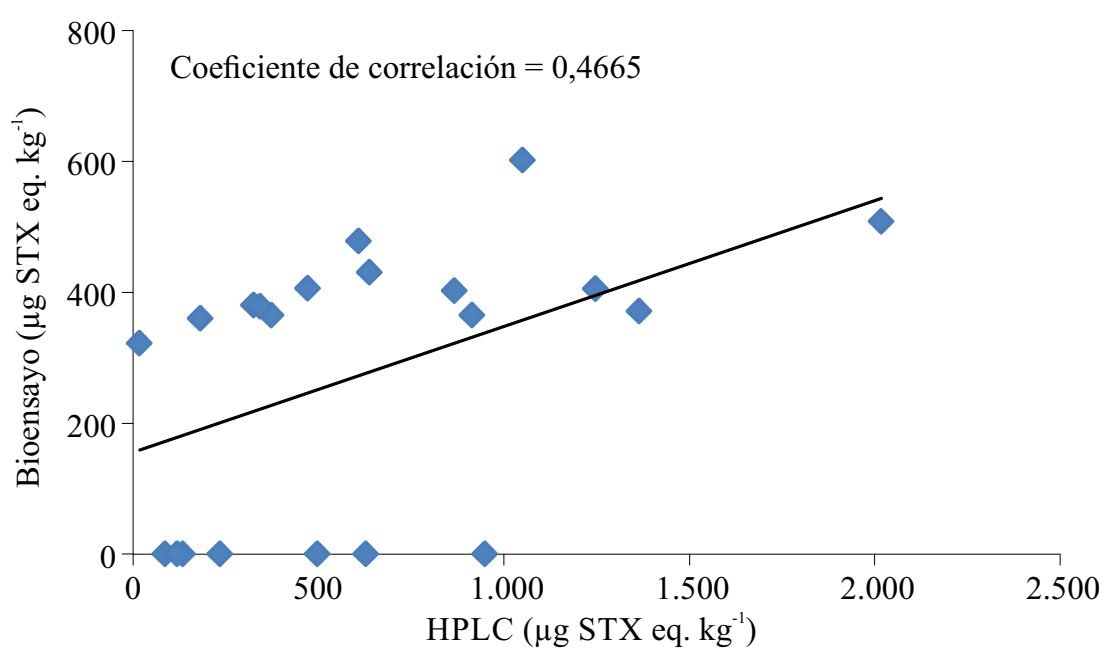

Figura 9. Correlación entre los resultados de los análisis de toxicidad (toxina paralizante de moluscos) obtenidos mediante bioensayo y HPLC.

Figure 9. Correlation among the results of the toxicity analyses (paralyzing shellfish toxin) obtained through bioassay and HPLC. 

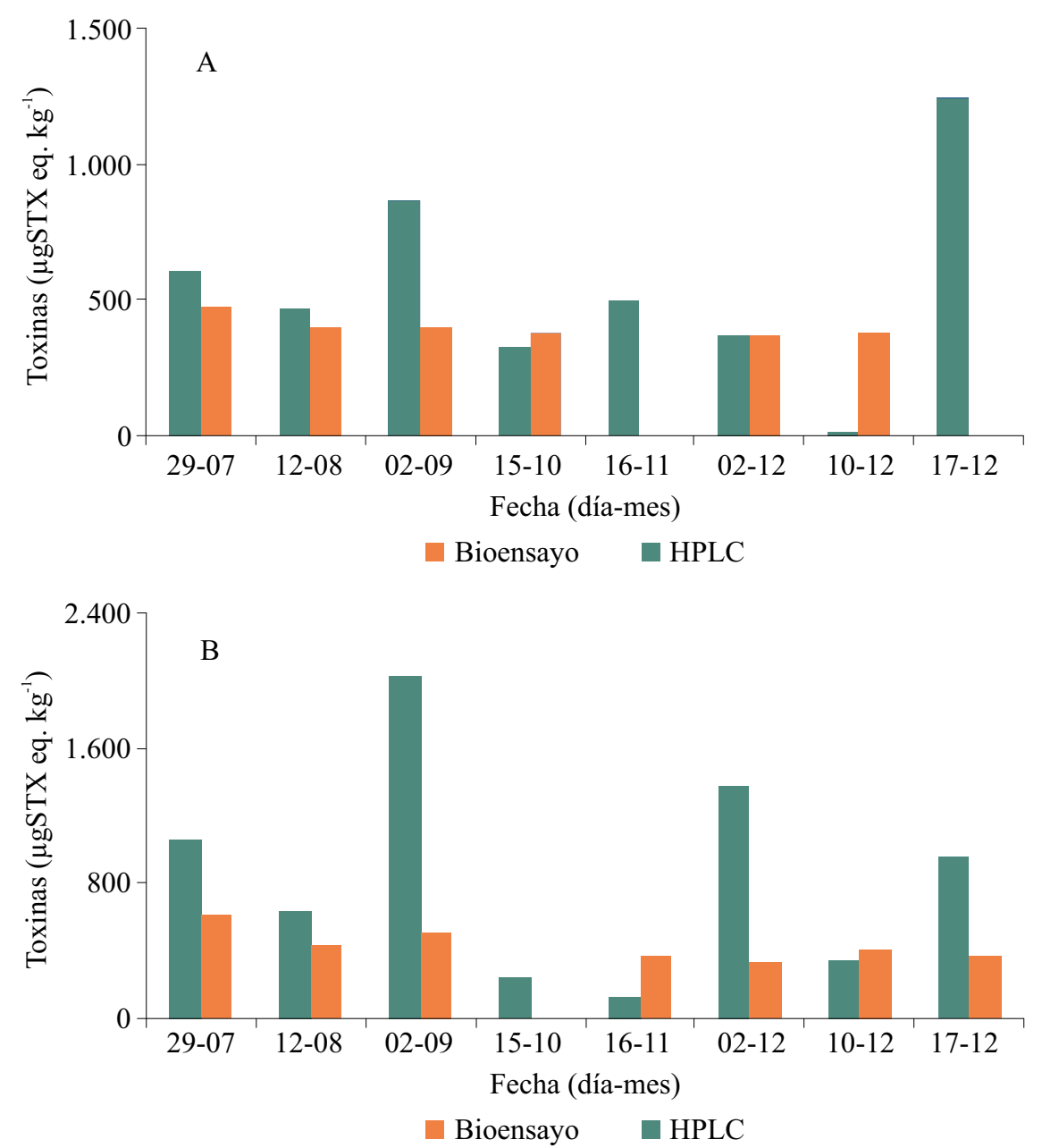

Figura 10. Toxinas paralizantes de moluscos expresadas como toxicidad determinadas mediante bioensayo y HPLC en mejillones (A) y cholgas (B) en la entrada de Bahía A. Brown (estación 6) obtenidas desde el 29 de julio al 16 de diciembre de 2013.

Figure 10. Paralyzing shellfish toxins expressed as toxicity determined through bioassay and HPLC in mussels (A) and cholgas (B) at the entrance of A. Brown Bay (station 6) collected from July $29^{\text {th }}$ through December $16^{\text {th }} 2013$.

que podría indicar una incorporación más reciente de las toxinas, pues en el inicio del evento tóxico los mejillones mantienen el perfil del dinoflagelado productor, pero luego los procesos de transformación metabólica o enzimática causan la variación del perfil tóxico (Oshima 1995).

Aunque se registraron densidades significativas de las diatomeas Pseudo-nitzschia spp., las muestras de cholgas y de mejillones no presentaron toxina amnésica de moluscos.

\section{Análisis de pigmentos, MAAs y toxinas en las especies tóxicas aisladas}

Dos especies de dinoflagelados tóxicos, $A$. ostenfeldii y $A$. cf. tamarense aisladas previamente del sector de estudio dentro del Canal Beagle, fueron mantenidas en cultivo con el objetivo de estudiar sus características específicas y comparar la producción de MAAs, su perfil pigmentario y su composición de toxinas. 
A

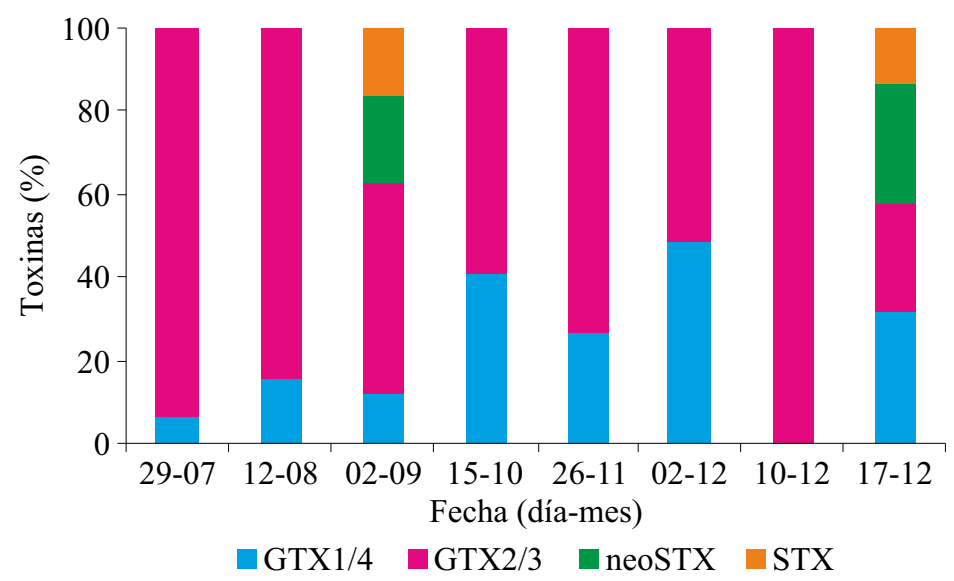

B

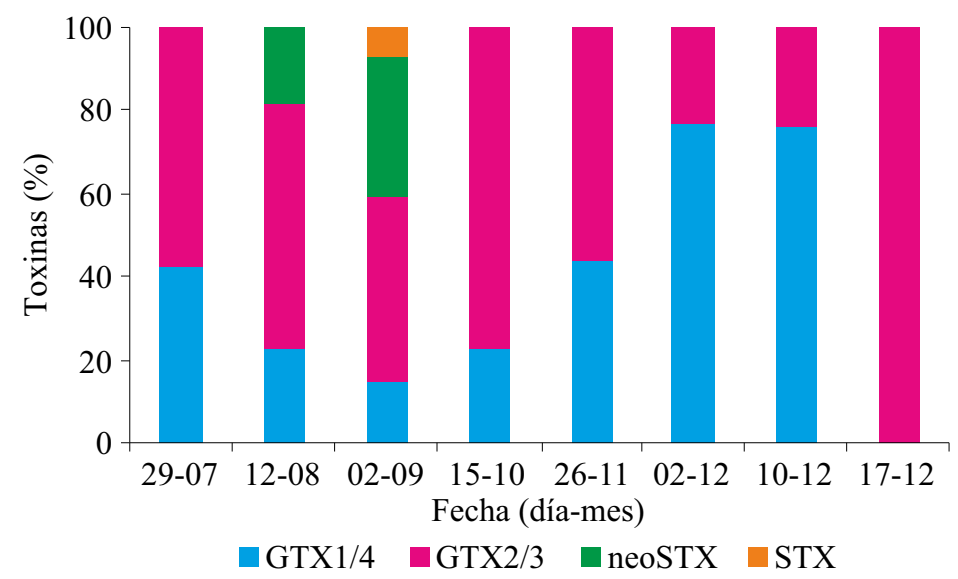

Figura 11. Toxinas detectadas en mejillones (A) y cholgas (B) en la entrada de Bahía Brown (estación 6) reunidas desde el 29 de julio al 16 de diciembre de 2013. GTX14 es la suma de los epímeros GTX1 y GTX4; GTX23 es la suma de los epímeros GTX2 y GTX3 (modificado de Montoya 2018).

Figure 11. Toxins detected in mussels (A) and cholgas (B) at the entrance of A. Brown Bay (station 6) collected from July $29^{\text {th }}$ through December 16 ${ }^{\text {th }}$ 2013. GTX14 is the sum of the GTX1 and GTX4 epimers; GTX23 is the sum of the GTX2 and GTX3 epimers (modified from Montoya 2018).

La concentración de clorofila $a$ en $A$. ostenfeldii fue 15 pg cél. ${ }^{-1}$, mientras que la de $A$. cf. tamarense fue tres veces mayor $\left(46,4\right.$ pg cél..$\left.^{-1}\right)$. $A$. ostenfeldii presentó la menor concentración de pigmentos totales por célula $(32,26$ pg cél.-1), siendo para $A$. cf. tamarense $87,82 \mathrm{pg}$ cél. ${ }^{-1}$. El perfil de pigmentos hallado en los dos cultivos fue similar y corresponde al característico de dinoflagelados, con peridinina como carotenoide principal seguido por clorofila $c_{2}$, y el grupo de carotenoides diadinoxantina, diatoxantina, dinoxantina, $\beta$ caroteno y perididinol (Figura 12).

Se encontraron diferencias importantes en la cantidad y el tipo de toxinas halladas en las dos especies de dinoflagelados aislados. A ostenfeldii produce principalmente toxinas del tipo espirólido (Almandoz et al. 2014) y solo se detectaron trazas de toxinas del grupo de TPM. El contenido total de TPM en $A$. ostenfeldii fue de 0,27 fmol cél. ${ }^{-1}$, mientras que en $A$. cf. tamarense fue de 18,8 fmol 


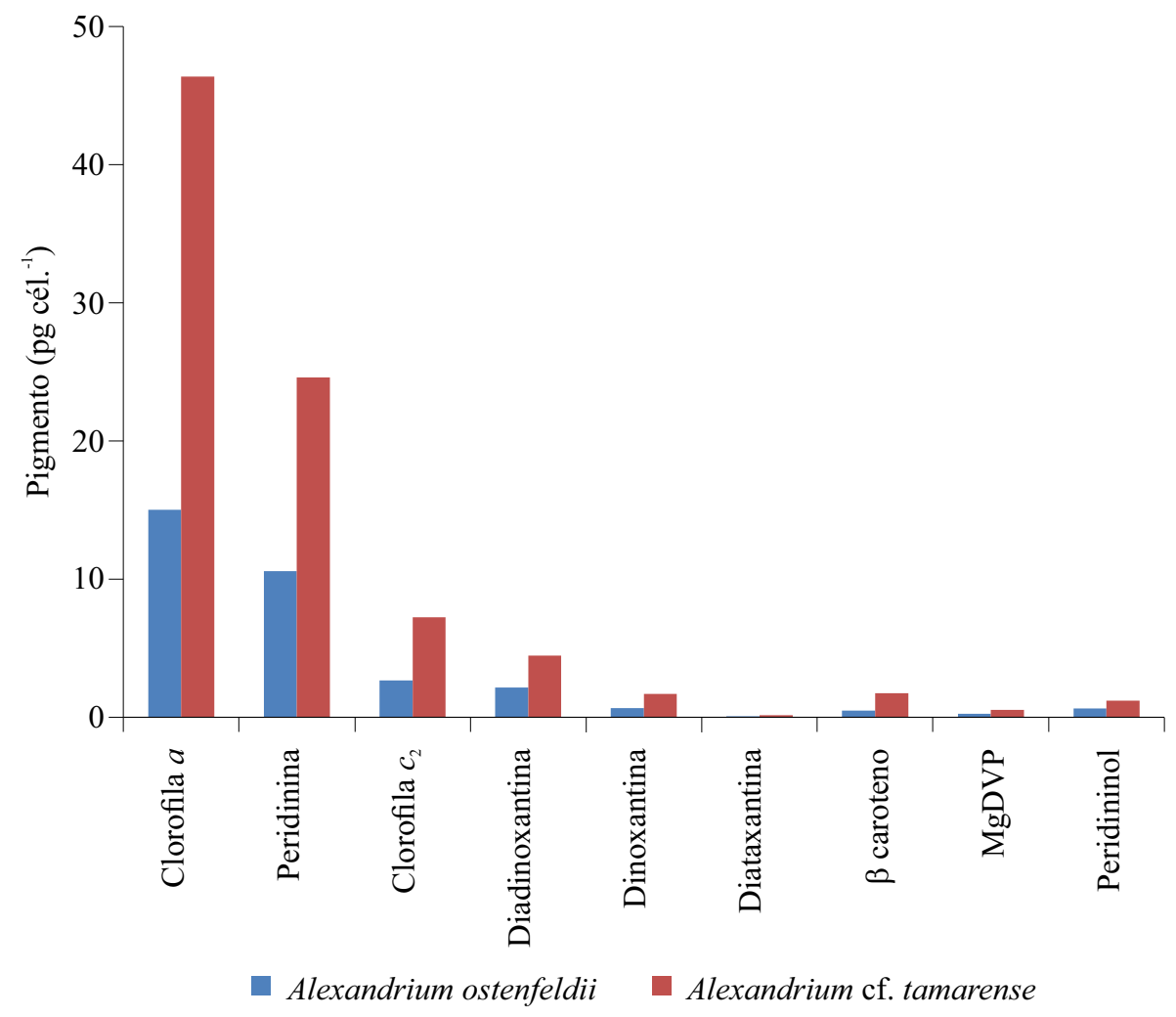

Figura 12. Concentración de pigmentos en los cultivos de Alexandrium cf. tamarense y A. ostenfeldii aislados del Canal Beagle. Figure 12. Pigments concentration in Alexandrium cf. tamarense and A. ostenfeldii cultures isolated from the Beagle Channel.

cél. ${ }^{-1}$ (70 veces más). En cuanto al perfil de toxinas en $A$. ostenfeldii solo se detectaron bajas concentraciones de GTX2/3, mientras que el perfil de $A$. cf. tamarense fue más complejo y similar al detectado para cultivos de $A$. tamarense aislados de Península Valdés (Montoya et al. 2010), con máximas concentraciones de GTX4 y C2 seguido de C1, GTX3 y GTX2 (Tabla 5). La toxicidad expresada como pg STX eq. cél. ${ }^{-1}$ fue de 11,83 para $A$. cf. tamarense y de 0,66 para $A$. ostenfeldii.

La concentración de MAAs totales en A. ostenfeldii alcanzó 4,97 fg cél. ${ }^{-1}$, siendo su composición en MAAs individuales relativamente menos compleja que la de otras especies de Alexandrium (Carreto et al. 2001). Se identificaron y cuantificaron 6 MAAs, siendo las más abundantes shinorine y palythene $\left(1,66\right.$ y $2,06 \mathrm{fg}$ cél. ${ }^{-1}$, respectivamente). Las 4 MAAs restantes (palythine, shino- rine methyl ester, porphyra-334 y mycosporine glycine presentaron valores de concentración semejantes, comprendidos en el rango de 0,20 a 0,43 fg cél. $^{-1}$ (Figura 13).

La composición en MAAs individuales de $A$. cf. tamarense fue muy similar a la registrada para otras especies de Alexandrium estudiadas previamente (Carreto et al. 2001), identificándose hasta 10 diferentes MAAs: shinorine, palythine, porphyra-334, mycosporine-glycine, acido zpalythenico, shinorine methyl ester, usujirene, palythene y los MAAs complejos M320 y M335/360 (Figura 13). Shinorine y palythene aparecen también como los más abundantes $(5,20$ y 6,92 fg cél. ${ }^{-1}$, respectivamente). También su concentración total de MAAs $\left(31,7 \mathrm{fg}\right.$ cél. $\left.^{-1}\right)$ fue notablemente superior ( $\sim 6$ veces $)$ a la determinada para $A$. ostenfeldii. 
Tabla 5. Concentración celular de toxinas (fmol cél. ${ }^{-1}$ ) paralizantes de moluscos en cultivos de Alexandrium cf. tamarense y A. ostenfeldii.

Table 5. Cell concentrations of paralytic shellfish toxins (fmol cel. ${ }^{-1}$ ) in cultures of Alexandrium $c f$. tamarense and A. ostenfeldii.

\begin{tabular}{lcc}
\hline Toxinas & Alexandrium cf. tamarense & Alexandrium ostenfeldii \\
\hline GTX4 & 9,85 & \\
GTX1 & 0,00 & \\
GTX3 & 0,25 & 0,09 \\
GTX2 & 0,04 & 0,18 \\
NeoSTX & 1,25 & \\
C1 & 0,89 & \\
C2 & 6,50 & 0,27 \\
\hline Total & 18,78 & \\
\hline
\end{tabular}

\section{DISCUSIÓN}

La explotación de mejillón (M. chilensis) en el área del Canal Beagle tiene un alto potencial de desarrollo debido a las óptimas condiciones agroecológicas que favorecen su comercialización (amplia disponibilidad del recurso y excelente calidad y tamaño de los mejillones) (Bertolotti et al. 2014). No obstante, la frecuencia de los eventos de toxicidad por microalgas nocivas, la diversidad de especies tóxicas que se presentan y las lentas velocidades de detoxificación de los moluscos en la región, son factores de riesgo importantes que deben ser evaluados para asegurar el apropiado manejo de esta pesquería.

Conocer las condiciones físicas del ambiente, tales como la turbulencia y la advección por mareas o por circulación de las masas de agua es esencial para la comprensión de la distribución vertical de los organismos, la formación de floraciones de microalgas nocivas y los patrones de toxicidad regionales. Tanto la dinámica de las poblaciones como la producción de toxinas pue- den ser moduladas en varias formas por los parámetros ambientales como temperatura, salinidad y disponibilidad de nutrientes (Reid 1997; Smayda y Reynolds 2001; Smayda 2002), por lo que es necesario conocer su distribución dentro de la bahía para evaluar las formas más apropiadas de manejo en los sistemas de cultivo de mejillón y reducir los riesgos sanitarios asociados a la ocurrencia de eventos tóxicos.

En el sector argentino del canal, la mayor parte de la producción del recurso proviene de la Bahía A. Brown, situada en la zona este del canal. La Bahía A. Brown tiene una superficie total de $7,37 \mathrm{~km}^{2}$ y una profundidad media de $11 \mathrm{~m}$ (Quiros et al. 1993). Presenta una entrada amplia (desde Bal. Dirección hasta Punta Gable), que conduce a una más cerrada de 1,65 km de ancho (entre Punta Almanza y Morro Gibraltar), donde la profundidad supera los $20 \mathrm{~m}$. Su parte más ancha tiene $2,6 \mathrm{~km}$ y su longitud es de aproximadamente $8,6 \mathrm{~km}$. La bahía no está internamente cerrada debido a que en su interior se comunica con las aguas del canal principal del Beagle al este de la Isla Gable mediante un brazo marítimo que contornea el borde norte de la isla, el Paso R. 

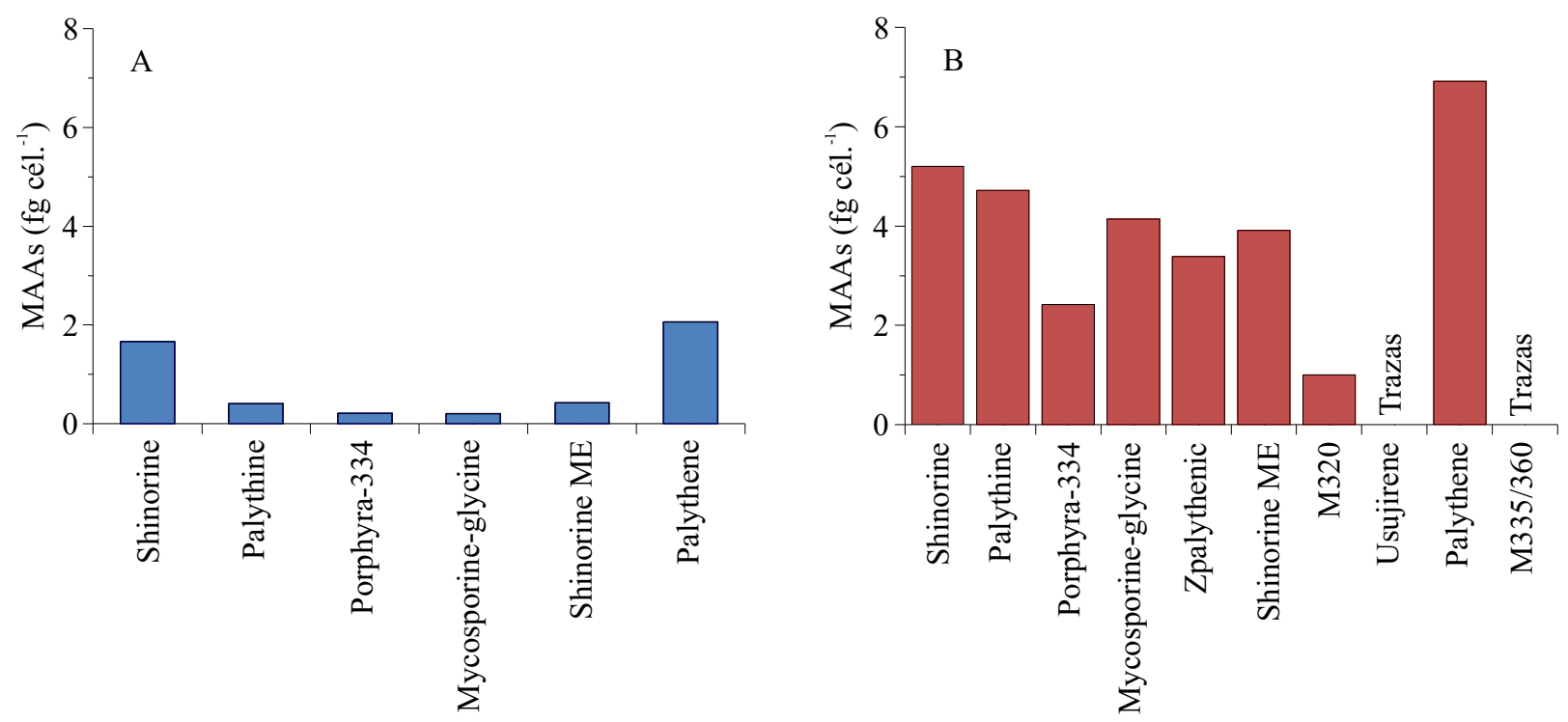

Figura 13. Concentración celular de MAAs individuales en cultivos de Alexandrium ostenfeldii y A.cf. tamarense.

Figure 13. Cell concentration of individual MAAs in Alexandrium ostenfeldii and A. $c f$. tamarense cultures.

Guaraní. Las mareas son del tipo "semidiurno", con marcada diferencia de amplitud entre las dos ondas sucesivas y una amplitud media de $1,20 \mathrm{~m}$ (Balestrini et al. 1998).

La circulación de las corrientes hidrográficas dentro de la Bahía A. Brown tiene características particulares debido a su condición de bahía "semicerrada". Sus aguas permanecen dentro durante el descenso de la marea y se renuevan durante su ascenso de Oeste hacia el Este, ingresando desde la zona de Punta Paraná y orientándose a través del paso Piedra Buena para luego pasar por el Paso R. Guaraní (Balestrini et al. 1998; Amin 1999). Amin (1999) registró el movimiento de agua dentro de la bahía mediante boyas de deriva y observó que la corriente no es suficiente para que las boyas liberadas en la zona central se dirijan hacia el final de la bahía (A. Brown), por lo que infiere que el recambio de agua se produce por diferencia de mareas más que por corrientes netas en la zona interior.

La distribución de los parámetros ambientales registrados en este estudio permitió diferenciar cuatro zonas dentro del área, con diferentes carac- terísticas hidrobiológicas determinadas principalmente por la circulación, los aportes de agua dulce y la profundidad (Figura 14):

1) La zona aledaña a la costa norte, desde la entrada hasta el sector central de la bahía, caracterizada por una micro-capa superficial de agua muy diluida por el aporte local proveniente del Río Almanza, que además determinó mayor aporte de nutrientes inorgánicos. Esta estructura vertical de dos capas es característica de los fiordos durante los meses de verano o primavera-otoño como consecuencia del deshielo (Isla et al. 1999), y se observó también en algunas bahías y accidentes costeros de otros sectores del canal donde el proceso de mezcla es restringido (Balestrini et al. 1998), y en algunos canales chilenos (Vera et al. 1996). La descarga de agua dulce continental en estas áreas costeras contribuye directamente la formación de una capa de mezcla superficial que tiene un pronunciado efecto sobre diferentes organismos planctónicos. La brusca disminución superficial de la salinidad 


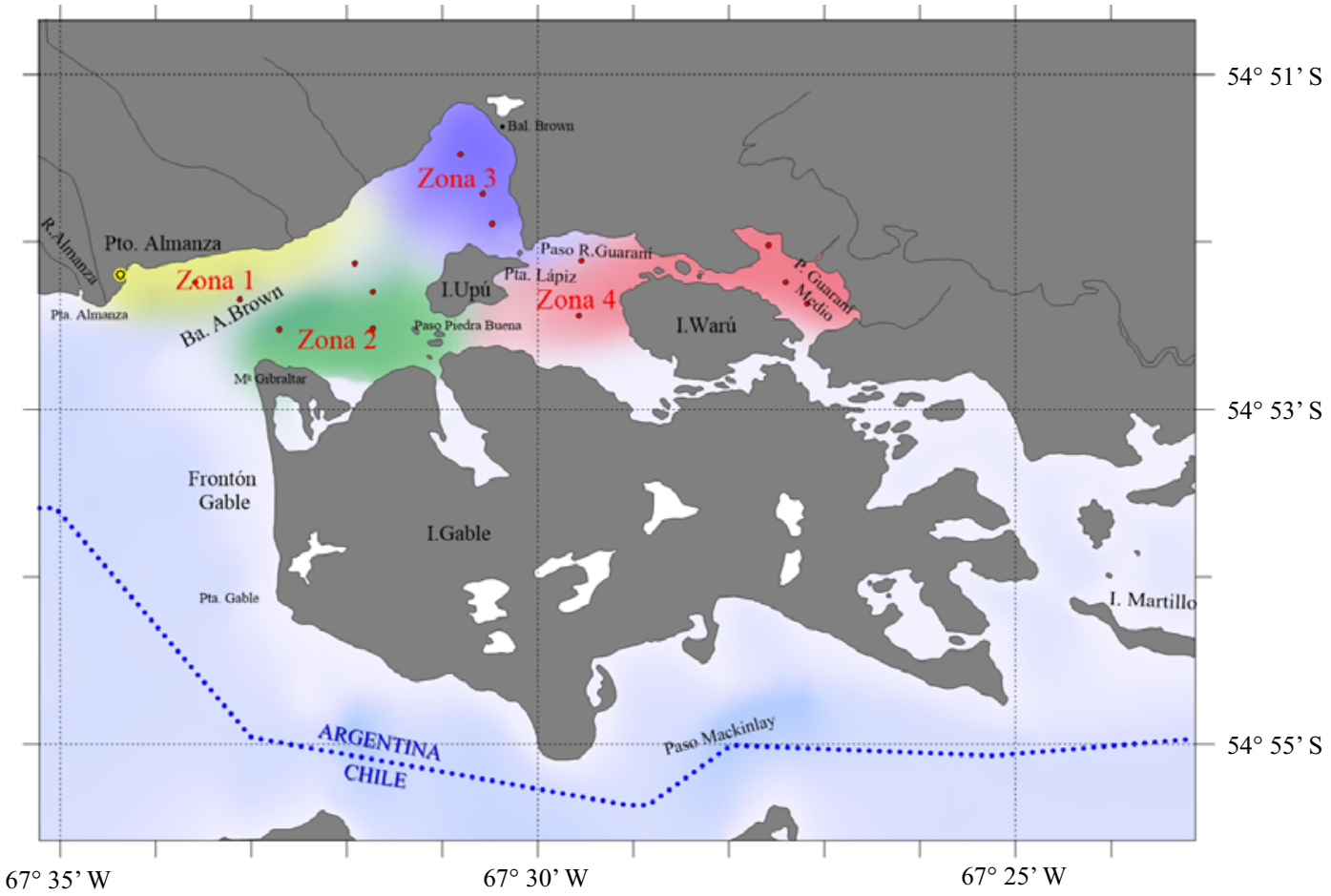

Figura 14. Distribución de las zonas hidrobiológicas diferenciadas en el área de Bahía A. Brown.

Figure 14. Distribution of the hydrobiological zones differentiated in the A. Brown Bay area.

produce una marcada estratificación salina que disminuye la turbulencia, que es un factor crítico para el florecimiento de los flagelados (Margalef 1978). La estabilidad de la columna de agua inducida por la estratificación salina es una de las condiciones que favorecen el crecimiento de dinoflagelados del Género Dinophysis, como fue observado por Peperzak et al. (1996) para los florecimientos de D. acuminata en el Mar del Norte. Los florecimientos de esta especie en la región de Antifer, Francia, fueron también relacionados con la marcada disminución de salinidad y aumento de nitratos causada por el desplazamiento superficial de aguas estuariales del Sena (Lassus et al. 1993). En los fiordos del sur de Chile, el crecimiento y acumulación del fitoplancton fueron asociados a un aumento de la estabilidad vertical y estratificación superficial de la columna de agua debido a la fusión de los campos de hielos continentales (Pizarro et al. 2000; Avaria 2008).

2) La zona aledaña a la costa de Isla Gable, desde la entrada hasta el sector central de la bahía, con una casi completa homogeneidad vertical de los parámetros físicos y salinidades mayores de 30,6 en toda la columna de agua. La mayor salinidad y la ausencia de estructura vertical indican que la zona está más relacionada al flujo central del canal (Isla et al. 1999), que tiene mayor incidencia sobre este sector de la bahía debido a la barrera geográfica ofrecida por la Isla Gable.

3) La zona interior de la bahía, donde la circulación restringida permitió el desarrollo parcial de una estructura vertical en la distribución de los parámetros físicos. El desplazamiento restringido de agua registrado en el interior de la bahía determina las características hidrobiológicas particulares de este sector, que puede ser 
considerado como un área de retención donde la baja velocidad y el intercambio reducido favorecen la sedimentación de partículas de menor granulometría y el desarrollo incipiente de una estructura vertical en la distribución de los parámetros físicos. Estudios previos sobre la composición granulométrica de los sedimentos en el sector interior de la bahía mostraron una alta proporción (30-40\%) de limo-arcillas. A diferencia de los sedimentos del sector central y sector costero, presentó una composición mayoritaria de arenas (Amin 1999). Las áreas con circulación restringida, donde prevalece la sedimentación sobre el transporte, pueden funcionar como trampas de sedimentos y por lo tanto reservorios potenciales de estadíos bentónicos de algunos dinoflagelados. Las hipnocigotas de resistencia producidas por muchas especies de dinoflagelados tienden a concentrarse en sedimentos finos más que en los gruesos arenosos debido a que tienen un comportamiento hidrodinámico similar al de las partículas de limo (Dale 1976; Angles et al. 2010), dando lugar a la formación de "bancos" de quistes. La presencia de estos bancos tiene una considerable significación biológica debido a que la germinación de los quistes proporciona un inóculo inicial para la formación de florecimientos de dinoflagelados o la producción de eventos locales de toxicidad (Anderson y Wall 1978; Anderson 1984), por lo cual, el monitoreo de las poblaciones bentónicas de quistes de dinoflagelados y la localización de las áreas que actúan como reservorios de estos quistes proporcionan una información importante que puede proveer una alerta temprana sobre la presencia y abundancia de especies tóxicas en éstas áreas.

4) La zona del Paso R. Guaraní, donde la escasa profundidad favoreció el incremento de la temperatura y la penetración de la radiación solar hasta el fondo. La gran disponibilidad de luz sobre el fondo en este sector proporciona condiciones favorables para el crecimiento de espe- cies bentónicas de fitoplancton como Prorocentrum lima. Esta especie, productora de toxinas lipofílicas, tiene un hábito principalmente bentónico o epifítico (Faust 1991); y fue observada en las muestras de fitoplancton tomadas en el lugar, aunque en baja abundancia.

El predominio de dinoflagelados comúnmente registrado a principios de diciembre en el área del Canal Beagle está caracterizado por la presencia de especies productoras de toxina paralizante de moluscos (TPM) como A. catenella y A. tamarense (Benavides et al. 1995; Guzmán et al. 2002; Almandoz et al. 2011), lo cual se evidencia en los niveles de toxicidad de moluscos bivalvos registrados en los monitoreos de toxinas realizados rutinariamente en el área (Goya y Maldonado 2014). La inusual ausencia de toxicidad registrada en diciembre de 2013 fue coincidente con una escasa presencia de dinoflagelados observada en el plancton y la ausencia de las especies de Alexandrium mencionadas, las cuales normalmente causan el incremento de toxicidad en esta época del año en la Bahía A. Brown. La ausencia de estas especies de dinoflagelados y la dominancia del Género Chaetoceros en el plancton mostraron que la comunidad de fitoplancton se hallaba en una etapa temprana de desarrollo, indicando un retraso en la típica secuencia sucesional descripta por Margalef (1978), que comienza a principios de primavera con el florecimiento de diatomeas pequeñas formadoras de cadenas y de rápido crecimiento, como la mayoría de las especies del género Chaetoceros, seguida por el crecimiento de diatomeas de mayor tamaño. Florecimientos de Chaetoceros han sido registrados previamente en otros sectores del Canal Beagle durante la primavera temprana (Avaria et al. 2003; Pizarro et al. 2005). Almandoz et al. (2011) estudiaron la variación estacional de la composición del fitoplancton en área de Bahía A. Brown durante un ciclo anual y observaron que el incremento en la biomasa y densidad celular registrado a principios de pri- 
mavera está asociado al florecimiento de varias especies de diatomeas del Género Chaetoceros, entre las que presentan mayor abundancia $C$. socialis, $C$. tortissimus, $C$. debilis y $C$. decipiens. Igualmente, la única especie de Alexandrium observada en las muestras tomadas en este período (A. ostenfeldii) es normalmente observada en el plancton durante el mes de octubre, en coincidencia con el inicio del florecimiento primaveral de las diatomeas, causando un pico menor de toxicidad en los moluscos bivalvos del área en ese período (Hernando com. pers.) ${ }^{1}$.

De acuerdo con el estudio realizado por Almandoz et al. (2011), el florecimiento de Chaetoceros es seguido por un pico menor de abundancia representado por el Género Thalassiosira, después del cual la biomasa de diatomeas decrece, incrementándose la abundancia de los dinoflagelados sobre el final de la primavera. La composición específica del fitoplancton durante diciembre de 2013 estuvo dominada en por diatomeas del Género Chaetoceros. Esta composición, y la ausencia de toxicidad en este período, sugieren que la comunidad fitoplanctónica se hallaba en una etapa temprana de la secuencia sucesional clásica.

Las diferencias observadas con estudios previos en la composición específica de fitoplancton en el área estudiada pueden estar relacionadas a la baja temperatura y velocidad del viento registradas por la estación meteorológica del aeropuerto de la ciudad de Ushuaia durante los meses de primavera previos. Coincidentemente, en el Boletín Climatológico estacional publicado por el Servicio Meteorológico Nacional, se registraron anomalías térmicas negativas a partir de la primavera de 2013 hasta enero de 2014, principalmente en el centro y sur de la Patagonia (SMN, 2013), siendo el verano 2013/2014 el más frio de las últimas cinco décadas. Otra anomalía registrada por el SMN durante la primavera de 2013 fue que las frecuencias de días con cielo cubierto fueron marcadamente positivas en la región. Estas anomalías atmosféricas podrían haber sido uno de los factores que causó el retraso en el desarrollo de la secuencia sucesional usual del fitoplancton en la región, alterando la composición específica esperada para este período del año, lo cual pone de manifiesto la necesidad de realizar un monitoreo frecuente de los parámetros atmosféricas regionales para favorecer la interpretación de la variabilidad observada en las comunidades de fitoplancton.

Las fracciones de nano y picoplancton son importantes componentes del fitoplancton en los océanos y carecen en algunos casos de caracteres morfológicos definitorios, por lo que su estudio requiere técnicas especiales. Estos organismos, presentan un perfil complejo de pigmentos fotosintéticos (clorofilas, carotenoides y ficobilinas) distribuidos selectivamente en diferentes taxones (Jeffrey et al. 1997). La presencia de pigmentos marcadores propios de diferentes taxones, generalmente a nivel de clase y excepcionalmente al nivel de género y especie, es una ayuda importante para caracterizar la composición de la comunidad de fitoplancton en muestras naturales. En las muestras analizadas, se identificaron componentes del nano y picoplancton según su perfil pigmentario, observándose una muy buena correlación entre los pigmentos determinados y los grupos fitoplanctónicos observados en microscopio, siendo el primer estudio de este tipo que se realiza en Bahía A. Brown.

Los niveles de clorofila determinados corresponden a una etapa inicial del crecimiento fitoplanctónico primaveral. La presencia de altas concentraciones de fucoxantina, la baja diversidad pigmentaria y el incremento en el nivel de clorofila $a$ del 10 al 17 de diciembre, se corresponden con el crecimiento de diatomeas observado y es coincidente con la disminución de nutrientes registrada entre ambos muestreos.

\footnotetext{
${ }^{1}$ Marcelo Hernando, Departamento de Radiobiología, Comisión Nacional de Energía Atómica, Av. Gral. Paz 1499, B1650KNA

- Buenos Aires, Argentina.
} 


\section{Perfil de toxinas y toxicidad}

En la región se han registrado varias especies de diatomeas del Género Pseudo-nitzschia ( $P$. australis, $P$. calliantha, $P$. fraudulenta y $P$. cf. seriata) potenciales productoras de ácido domoico (TAM) (Almandoz et al. 2011). Sin embargo, en el canal solo se ha detectado AD en una campaña realizada en abril de 2012 en la que no se identificó la especie productora (Krock et al. 2015). La ausencia de AD en las muestras estudiadas puede deberse a diversos factores. El primer factor a considerar es una baja concentración de células o bajo nivel de toxinas por célula y por lo tanto un nivel de toxinas no detectable. Otro punto a tener en cuenta es que la producción de toxinas por el Género Pseudo-nitzschia depende de múltiples factores tanto externos (nutrientes, luz, hierro, cobre, etc.) como internos (fase de crecimiento, endobacterias, etc.) (Lelong et al. 2012). La presencia de una especie potencialmente tóxica en el plancton no es sinónimo de toxicidad, pero es un alerta, y por lo tanto es necesario realizar los estudios correspondientes para verificar su capacidad como productor de toxinas y monitorear los posibles organismos afectados.

El análisis oficial de Toxinas Paralizantes de Moluscos en la Argentina se realiza por el método tradicional de bioensayo con ratones (SAGPYA 2006). Sin embargo, por razones éticas en diversas legislaciones, como en la Comunidad Europea, no se considera éste un método adecuado. El uso potencial de técnicas analíticas para reemplazar bioensayos con ratón está siendo discutido, para lo cual los métodos que utilizan HPLC resultan los más adecuados. El método analítico permite conocer y cuantificar cada una de las toxinas que forman el grupo de TPM, y por lo tanto es el ideal para estudiar los fenómenos de transferencia y metabolización en los diferentes organismos afectados. Sin embargo, presenta algunas desven- tajas en los programas de monitoreo cuando es necesario controlar en forma rápida una gran cantidad de muestras (Etheridge 2010). Frente a la rápida y segura respuesta que brinda el bioensayo, el método oficial de análisis por HPLC propuesto por la Comunidad Europea tiene el inconveniente de la interpretación de los resultados, la necesidad de contar con todos los estándares y el tiempo insumido en la extracción, purificación y análisis de las muestras (EFSA 2009). Frente a este problema, una solución que se está evaluando es la utilización de kits rápidos para el análisis rutinario en los programas de monitoreo (Etheridge 2010).

El análisis de toxinas por HPLC en muestras de bivalvos del mismo período mostró la presencia de TPM en muy bajas concentraciones, por lo que es de suponer que en el período estudiado no hubo eventos extraordinarios de especies toxicas de fitoplancton. Con el objetivo de estudiar la evolución temporal de la toxicidad, se analizaron muestras de mejillones y cholgas correspondientes a un período anterior. En el análisis de las muestras colectadas en el período julio a diciembre de 2013, si bien algunas muestras fueron negativas por bioensayo, en todas fue posible detectar TPM en diferente concentración utilizando el método más sensible de HPLC. El bajo coeficiente de correlación observado entre los métodos puede deberse al error típico del bioensayo (20-30\%), a que la sensibilidad del análisis por HPLC es superior, o a la presencia de sustancias que son toxicas para los ratones y no son detectadas por el método de HPLC (Costa et al. 2009). La baja presencia de especies tóxicas en el plancton y la detoxificación de los moluscos observada en el período abarcado por este estudio, sugieren que las toxinas fueron adquiridas en un evento tóxico previo ocurrido en el verano de 2012-2013 (Eriksson com. pers.) ${ }^{2}$. Sin embargo, tanto en mejillones como en cholgas, se observó un incremento del contenido total

\footnotetext{
${ }^{2}$ Nahuel Eriksson, Secretaría de Ambiente, Desarrollo Sostenible y Cambio Climático de la Provincia de Tierra del Fuego, Antártida e Islas del Atlántico Sur, San Martín 1401 - Ushuaia, Argentina.
} 
de toxinas en las muestras tomadas el 2 de septiembre y 17 de diciembre. Si bien no se observa un incremento de las especies productoras de TPM en la comunidad fitoplanctónica, otros factores pueden haber causado esta variación, como la re-suspensión de las hipnocigotas de dinoflagelados tóxicos. Se ha atribuido a las hipnocigotas la presencia de toxicidad en algunos filtradores (almeja y culengue) de la Región de Aysén, reportada durante el invierno, en ausencia de la fase móvil (Lembeye 1998). Otro factor posible en la variación del perfil de toxinas en las muestras de bivalvos es la diferente composición de toxinas en los dinoflagelados A. catenella y A. ostenfeldii, cuya sucesión en el plancton podría reflejarse como un cambio en el perfil tóxico en las primeras etapas de intoxicación de los mejillones. Además, los cambios fisiológicos de estadio y madurez sexual de los bivalvos pueden modificar el contenido de humedad y grasas total de la muestra, con el consecuente cambio en la cantidad relativa de toxinas expresadas en peso húmedo (Bricelj $\mathrm{y}$ Shumway 1998). En la región de Canal Beagle, el tiempo de detoxificación de los mejillones puede ser mayor como consecuencia de la baja temperatura del agua, lo que ocasiona una baja velocidad de metabolización de las toxinas adquiridas. Por ejemplo, Álvarez (2012) observa un evento de TPM ocurrido en octubre de 2009 en Canal Beagle que impuso una veda que se extendió por todo un año. Otro factor importante en la velocidad de detoxificación es la concentración inicial de toxinas que suele ser elevada en los eventos registrados en el Canal Beagle (Goya y Maldonado 2014). Por otra parte, la diferencia de toxicidad entre mejillones y cholgas obtenidas en cultivo o en bancos naturales se ha observado en otros estudios y puede ser debida a diferencias en la metabolización enzimática de las toxinas (Bricelj y Shumway 1998).

El cultivo de $A$. ostenfeldii y $A$. cf. tamarense permitió realizar estudios controlados para profundizar el conocimiento de algunas de las características ecofisiológicas específicas como la pro- ducción de MAAs, el perfil pigmentario y la composición de toxinas. Los resultados obtenidos en este estudio demuestran que $A$. ostenfeldii, aislado del Canal y creciendo en condiciones óptimas de cultivo, solo produce muy baja cantidades de GTX2/3. Por otra parte, se ha observado que $A$. tamarense, aislado de la plataforma bonaerense, presenta mayor toxicidad creciendo en medio natural que en cultivo debido a las diferencias en el perfil toxico que presenta el dinoflagelado creciendo en condiciones diferentes (Montoya et al. 2010). Es por lo tanto importante estudiar la producción de toxinas de $A$. ostenfeldii en medio natural con el fin de comparar las características ecofisiológicas. El cultivo de $A$. cf. tamarense aislado en un estudio previo en el Canal Beagle presenta un perfil de toxinas similar al de $A$. tamaren$s e$ aislado de la plataforma bonaerense (Montoya et al. 2010). Igualmente, el resultado del análisis de pigmentos de los cultivos aquí estudiados fue coincidente con el esperado para dinoflagelados de este género (Jeffrey et al. 1997), presentando peridinina como pigmento característico.

Los efectos nocivos que inducen la radiación UV en organismos acuáticos pueden ser aminorados a través de varios mecanismos de fotoprotección entre los que se encuentran, además, la activación de sistemas antioxidantes, la acumulación de sustancias que absorben radiación UV (fotoprotectores). Entre los fotoprotectores se encuentran los aminoácidos tipo micosporina (MAAs). Los dinoflagelados han desarrollado la habilidad de sintetizar este tipo de compuestos, lo que sumado a su capacidad de migración vertical en la columna de agua le confiere una decisiva ventaja adaptativa. En A. ostenfeldii la composición en MAAs individuales fue relativamente menos compleja que la de otras especies de Alexandrium, mientras que en $A$. cf. tamarense fue muy similar a las estudiadas previamente (Carreto et al. 2001). Dado que la disminución de la capa de ozono en la región es máxima en primavera, sería conveniente realizar en un futuro estudios de fotoadaptación y presencia de aminoácidos simi- 
lares a micosporinas en estas especies en medio natural.

En 2016, las floraciones de Pseudochattonella causaron una mortalidad del $20 \%$ de la producción total de salmón chileno: en unos pocos días se sacrificaron unos 30 millones de peces (Clement et al. 2016). Estos eventos fueron seguidos por floraciones excepcionales de A. catenella, que devastaron la producción de mariscos y llevaron a una agitación social que afectó gran parte de la zona productiva. Estos fenómenos se asociaron claramente con anomalías climáticas y se vincularon a una de las señales más fuertes de El Niño en el Océano Pacífico Sudoriental en las últimas décadas (Muñoz et al. 2018). La experiencia obtenida en los últimos años sobre los eventos de toxicidad nos ha demostrado que se debe prestar especial atención al advenimiento de nuevas especies tóxicas y a la ocurrencia de nuevos síndromes tóxicos en la región. Así mismo, se advierte la necesidad de realizar un monitoreo microscópico de los sedimentos bentónicos en el sector interno de la Bahía A. Brown junto con el de las especies tóxicas presentes en el plancton, así como contar con un registro continuo de las variables meteorológicas para intentar desarrollar una capacidad predictiva de los florecimientos de algas nocivas en el canal.

\section{AGRADECIMIENTOS}

Agradecemos la colaboración de la Subsecretaría de Desarrollo Sustentable y Ambiente y la Dirección General de Desarrollo Pesquero y Acuícola del Gobierno de la Provincia de Tierra del Fuego, Antártida e Islas del Atlántico Sur para la realización los muestreos. Al Sr. Nahuel Erikkson, director del Laboratorio Ambiental de la SADSyCC por facilitar las instalaciones para los análisis preliminares de las muestras. A la Sra. Belén Mattera por su colaboración en los análisis de pigmentos. Contribución INIDEP N 2191.

\section{REFERENCIAS}

Almandoz Go, Fabro E, Ferrario M, Tillmann U, Cembella A, Krock B. 2017. Species occurrence of the potentially toxigenic diatom genus Pseudo-nitzschia and the associated neurotoxin domoic acid in the Argentine Sea. Harmful Algae. 63: 45-55.

Almandoz GO, Hernando MP, Ferreyra GA, Schloss IR, FERrario ME. 2011. Seasonal phytoplankton dynamics in extreme southern South America (Beagle Channel, Argentina). J Sea Res. 66 (2): 47-57.

Almandoz GO, Montoya N, Hernando MP, Benavides H, Carignan M, Ferrario ME. 2014. Toxic strains of the Alexandrium ostenfeldii complex in South America (Beagle Channel, Argentina). Harmful Algae. 37: 100109.

Álvarez M. 2012. Situación actual de las zonas de producción de moluscos bivalvos en referencia a los fenómenos de Marea Roja. Años 2008 al 2011. $5^{\text {to }}$ Taller de Trabajo de la Red de Fortalecimiento para la Maricultura Costera Patagónica y del Taller de Cultivo de Mejillón. <http://www. cenpat-conicet.gov.ar/ mariculturaenred/PresentacionesTaller/Mareas rojas-FerinoAlvarez.pdf $>$.

Amin OA. 1999. Estudio de las características oceanográficas de la Bahía Almirante Brown. Informe para la Subsecretaría de Recursos naturales y Ambiente Humano de la prov. de Tierra del Fuego, Antártida e Islas del Atlántico Sur, Argentina. 9 p.

ANDERSON DM. 1984. The roles of dormant cysts in toxic dinoflagellate blooms and shellfish toxicity. En: Ragelis E, editor. Seafood toxins. American Chemical Society Symposium series. Washington: American Chemical Society. p. $125-138$.

Anderson DM, Wall D. 1978. Potential importance of benthic cysts of Gonyaulax tamaren- 
sis and G. excavata in initiating toxic dinoflagellate blooms. J Phycol. 14: 224-234.

Angles S, Garces E, Jordi A, Basterretxea G, Palangues A. 2010. Alexandrium minutum resting cyst distribution dynamics in a confined site. Deep-Sea Res (II Top Stud Oceanogr). 57 (3-4): 210-221.

[AOAC] Association of Official Analytical Chemists. 1985. Paralytic shellfish poison. Biological method. Final action. Official Methods of Analysis. Sec. 959.08. p. 21-22.

AvariA, S. 2008. Phytoplankton in the austral Chilean channels and fjords. Progress in the oceanographic knowledge of Chilean interior waters, from Puerto Montt to Cape Horn. En: Silva N, Palma S, editores. Valparaíso: Comité Oceanográfico Nacional-Pontificia Universidad Católica de Valparaíso. p. 89-92.

Avaria S, Caceres C, Castillo P, Muñoz P. 2003. Distribución del microfitoplancton marino en la zona del Estrecho de MagallanesCabo de Hornos, Chile, en la primavera de 1998 (crucero CIMAR 3 fiordos). Cienc Tecnol Mar. 26: 79-96.

Balestrini C, Manzella G, Lovrich G. 1998. Simulación de corrientes en el Canal Beagle y Bahía Ushuaia mediante un modelo bidimensional. Servicio de Hidrografía Naval, República Argentina. Departamento Oceanografía. Informe Técnico $\mathrm{N}^{\circ} 98,58 \mathrm{p}$.

Benavides H, Prado L, Diaz S, Carreto Ji. 1995. An exceptional bloom of Alexandrium catenella in the Beagle Channel, Argentina. En: Lassus P, Arzul G, Erard E, Gentien P, Marcaillou C, editores. Harmful marine algal blooms. Proceedings of the Sixth International Conference on Toxic Marine Phytoplankton, October 1993, Nantes, France. p. 113-119.

Bertolotti Mi, Pagani A, Gualdoni P, Fosati J. 2014. Cadena de producción del cultivo del mejillón en el año 2011 en la provincia de Tierra del Fuego, Antártida e Islas del Atlántico Sur, Argentina. Rev Galega Econo. 23: 33-50.
Bricelu VM, Shumway SE. 1998. Paralytic shellfish toxins in bivalve molluscs: occurrence transfer kinetics and biotransformation. Rev Fish Sci. 6 (4): 315-383.

Carreto Ji, Carignan MO, Montoya NG. 2001. Comparative studies on mycosporine-like amino acids, paralytic shellfishtoxins and pigment profiles of the toxic dinoflagellates Alexandrium tamarense, A. catenella and A. minutum. Mar Ecol Prog Ser. 223: 49-60.

Carreto Ji, Carignan MO, Montoya NG. 2002. Short term effects of ultraviolet radiation on the toxic dinoflagellate Alexandrium catenella. Pigment bleaching and MAAs synthesis inhibition. In: Aquaculture, Environment and Marine Phytoplankton. G. Arzul Coord. Ed. IFREMER, Actes colloq. 34: 173-190.

Carreto Ji, Carignan MO, Montoya NG. 2005. A high-resolution reverse-phase liquid chromatography method for the analysis of mycosporine-like amino acids (MAAs) in marine organisms. Mar. Biol. 146: 237-252.

Cembella AD, Lewis NI, Quilliam MA. 2000. The marine dinoflagellate Alexandrium ostenfeldii (Dinophyceae) as the causative organism of spirolide shellfish toxins. Phycologia. 39: 67-74.

Clément A, Lincoqueo L, Saldivia M, Brito CG, Muñoz F, Fernández C, Pérez F, MaluJe CP, Correa N, Moncada V, Contreras G. 2016. Exceptional summer conditions and HABs of Pseudochatonella in Southern Chile create record impacts on salmon farms. Harmful Algae News. 53: 1-3.

Costa PR, Baugh KA, Wright B, Ralonde R, Nance SL, Tatarenkova N, Etheridge SM, Lefebvre KA. 2009. Comparative determination of paralytic shellfish toxins (PSTs) using five different toxin detection methods in shellfish species collected in the Aleutian Islands, Alaska. Toxicon. 54: 313-320.

DALE B. 1976. Cyst formation, sedimentation, and preservation: factors affecting dinoflagellate assemblages in recent sediments from Trond- 
heimsfjord, Norway. Rev Palaeobot Palynol. 22: 39-60.

[EFSA] European Food Safety Authority. 2009. Scientific opinion of the panel on contaminants in the food chain on is quest from the European Commission on marine biotoxins in shellfish-summary on regulated marine biotoxins. EFSAJ. 1306: 1-23.

ETHERIDGE SM. 2010. Paralytic shellfish poisoning: Sea food safety and human health perspectives Toxicon. 56: 108-122.

FAUST MA. 1991. Morphology of ciguatera-causing Prorocentrum lima (Pyrrophyta) from widely differing sites. J Phycol. 27: 642-648.

Ferrario ME, SAR EA, SAla S. 1995. Metodología básica para el estudio del fitoplancton con especial referencia a las diatomeas. En: Alveal K, Ferrario ME, Oliveira eC, Sar EA, editores. Manual de métodos ficológicos. Concepción: Universidad de Concepción. p. 1-23.

FRITZ L, TRIEMER RE. 1985. A rapid simple technique utilizing Calcofluor White M28 for the visualization of dinoflagellate thecal plates. J Phycol. 21: 662-664.

Goya AB, Maldonado S. 2014. Evolution of PSP toxicity in shellfish from the Beagle Channel (Tierra del Fuego, Argentina): an overview. En: SAUvÉ G, editor. Molluscan shellfish safety. Dordrecht: Springer. p. 15-23.

Grasshoff K, Ehrhardt M, KremLing K. 1983. Methods of sea water analysis. Weimnheim: Verlag Chemie. 419 p.

GuZman L, CAMPODÓNiCO E. 1975. Marea Roja en la Región de Magallanes. Publicaciones Instituto de la Patagonia. Serie Monografías, Punta Arenas, Chile, 9. 44 p.

GuZMAn L, LEMBEyE G. 1975. Estudios sobre un florecimiento tóxico causado por Gonyaulax catenella en Magallanes. II- Algunas condiciones hidrográficas asociadas. ANS. INST. PAT., Punta Arenas (Chile). VI (1-2): 185-195.

Guzman L, Pacheco H, Pizarro G, Alarcón C. 2002. Alexandrium catenella y veneno parali- zante de los mariscos en Chile. En: SAR EA, FERRARIO ME, REguERA B, editores. Floraciones algales nocivas en el cono sur americano. Vigo: Instituto Español de Oceanografía. p. 235-255.

Isla F, Bujalesky G, Coronato A. 1999. Procesos estuarinos en el Canal Beagle, Tierra del Fuego. Rev Asoc Geol Argent. 64 (4): 307-318.

Hydes D, Aoyama M, Aminot A, Becker S, Coverly S, Daniel A, Dickson A, Grosso O, Kerouel R, VAn OoiJen J, et AL. 2010. Determination of dissolved nutrients in seawater. En: Hood EM, SABINE CL, SLOYAN BM, editores. The GO-SHIP repeat hydrography manual: a collection of expert reports and guidelines. IOCCP Report No. 14, ICPO Publication Series No. 134. http://www. go-ship.org/HydroMan.html.

JEFFREY SW, WRIGHT SW. 1997. Qualitative and quantitative HPLC analysis of SCOR reference algal cultures. En: JEFFREY SW, MANTOURA RFC, WRIGHT SW, editores. Phytoplankton pigments in oceanography: guidelines to modern methods, Vol. 10. Paris: UNESCO Monographs on Oceanographic Methodology. p. 343-360.

Kremp A, Lindholm T, Dressler N, ERler K, Gerdts G, EirtovaAra S, Leskinen E. 2009. Bloom forming Alexandrium ostenfeldii (Dinophyceae) in shallow waters of the Åland Archipelago, Northern Baltic Sea. Harmful Algae. 8: 318-328.

Krock B, Borel M, Barrera F, Tillmann U, Fabrod E, Almandoz GO, Ferrario M, Garzón Cardona JE, Kocha BP, Alonso C, eT AL. 2015. Analysis of the hydrographic conditions and cyst beds in the San Jorge Gulf, Argentina, that favor dinoflagellate population development including toxigenic species and their toxins. J Mar Syst. 148: 86-100.

Lassus P, Proniewsiki F, Maggi P, Truquet P, BARDOUIL M. 1993. Wind-induced toxic blooms of Dinophysis cf. acuminata in the Antifer area (France). En: SMAYDA TJ, 
SHImizu Y, editores. Toxic phytoplancton blooms in the sea. Elsevier Science Publishers. p. 519-523.

Lelong A, Garet H, Soudant P, Bates S. 2012. Pseudo-nitzschia (Bacillariophyceae) species, domoic acid and amnesic shellfish poisoning: revisiting previous paradigms. Phycologia. 51 (2): 168-216.

LEMBEYE G. 1998. Seguimiento de la toxicidad en recursos pesqueros de importancia comercial en la X y XI Región, Informe Final Proyecto FIP. $86 \mathrm{p}$.

MARGALEF R. 1978. Life forms of phytoplankton as survival alternatives in an unstable environment. Ocenol Acta. 1: 493-509.

Montoya N, Carignan M, Benavides H. 2012. Toxinas emergentes: spirolidos en mejillones, fitoplancton y en cultivo de Alexandrium ostenfeldii del Canal de Beagle. Inf Invest INIDEP N 116/2012.

Montoya NG, Fulco KV, Carignan MO, CARRETO JI. 2010. Toxin variability in cultured and natural populations of Alexandrium tamarense from southern South America Evidences of diversity and environmental regulation. Toxicon. 56: 1408-1418.

Muñoz JL, Urbina MA, Garreaud R, Iriarte JL. 2018. Hydroclimatic conditions trigger record harmful algal bloom in western Patagonia (Summer 2016). Scientific Reports (2018) 8:1330. doi:10.1038/s41598-01819461-4

Nagai S, Nitshitani G, Tomaru Y, Sakiyama S, KamiYAma T. 2008. Predation by the toxic dinoflagellate Dinophysis fortii on the ciliate Myrionecta rubra and observation of sequestration of ciliate chloroplasts. J Phycol. 44 (4): 909-922.

Oshima Y. 1995. Post-column derivatisation liquid chromatography method for paralytic shellfish toxins. J AOAC Int. 78: 528-532.

Peperzak L, Snoeijer GJ, Dijkema R, Gieskes WWC, Joordens J, Peeters JCH, Vrieling EG, Zevenboom W. 1996. Development of
Dinophysis acuminata bloom in the river Rhine plume. En: Yasumoto T, Oshima Y, FUKUYO Y, editores. Harmful and toxic algal blooms. Intergovernmental Oceanographic Commission of UNESCO. 273-276.

Pizarro G, Iriarte, JL, Montecino V, Blanco JL, GuzMÁn L. 2000. Distribución de la biomasa fitoplanctónica y productividad primaria máxima de fiordos y canales australes $\left(47^{\circ}\right.$ $50^{\circ} \mathrm{S}$ ) en octubre 1996. Cienc Tecnol Mar. 23: 25-48.

Pizarro G, Montecino V, Guzman L, Muñoz V, Chacon V, Pacheco H, Frangopulos M, Retamal L, Alarcon C. 2005. Patrones anuales recurrentes del fitoplancton en fiordos y canales australes $\left(43^{\circ}-53^{\circ} \mathrm{S}\right)$ en primavera y verano. Cienc Tecnol Mar. 28 (2): 63-83.

Quiros R, Luchini L, Wicki G, ERrazti E. 1993. Evaluación e identificación de sitios aptos para el desarrollo de la acuicultura. Sobre la zona costera de la Isla Grande de tierra del Fuego y sus aguas interiores. Informe final para la Dirección de Recursos Naturales de la Prov. de Tierra del fuego Antártida e Islas del Atlántico sur. Consejo Federal de Inversiones de la República Argentina. Vol. I, II y II, 385 p.

REID PC. 1997. Discharges from hydroelectric power schemes as a trigger for marine algal blooms. Mar Pollut Bull. 34 (9): 730-733.

[SAGPYA] Secretaría de Agricultura, Pesca y Alimentación. 2006. Capitulo XXIII Productos de la Pesca 23.24 Reglamento Sanitario de explotación y comercialización de moluscos y bivalvos vivos para consumo humano directo. Resolución SAGPYA 829/2006. Decreto 4238/68.

[SMN] Servicio Meteorológico Nacional. 2013. Boletín climatológico trimestral. Primavera. Vol. XXV 2013. 13 p. http://www3.smn. gob.ar/serviciosclimaticos/clima/archivo/ clim-primav13.pdf.

SIMONSEN R. 1974. The diatom plankton of the Indian Ocean Expedition of R.V. "Meteor" 
1964-65. Meteor Forschungsergeb (D Biol). 19: 1-66.

SMAYDA, T.J. 2002. Turbulence, watermass stratification and harmful algal blooms: an alternative view and frontal zones as "pelagic seed banks". Harmful Algae. 1: 95-112.

Smayda TJ, ReYNOLDS CS. 2001. Community assembly in marine phytoplankton: application of recent models to harmful dinoflagellate blooms J Plank Res. 23 (5): 447-461.

Touzet N, Franco JM, Raine R. 2008. Morphogenetic diversity and biotoxin composition of Alexandrium (Dinophyceae) in Irish coastal waters. Harmful Algae. 7: 782-797.

UTERMÖHL H. 1958. Zur vervollkommung der quantitative phytoplankton-methodik. Mitt Int Ver Theor Angew Limnol. 9: 1-38.

Vera P, Avaria S, Muñoz P. 1996. Composición y distribución del fitoplancton de los fiordos adyacentes a Campos de Hielo Sur, Chile. Cienc Tecnol Mar. 19: 73-92.

Wright JLC, Quilliam MA, Cembella AD. 1995. Methods for domoic acid, the Amnesic Shellfish Poisons. En: HallegraefF GM, Anderson DM, Cembella AD, editores. Manual on Harmful Marine Microalgae, COI, Manual Guides $\mathrm{N}^{\circ}$ 33, UNESCO, Paris, $\mathrm{p}$ 113-133.

Zapata M, Rodriguez F, Garrido JL. 2000. Separation of chlorophylls and carotenoids from marine phytoplankton: a new HPLC method using a reversed phase $\mathrm{C} 8$ column and pyridine-containing mobile phases. Mar Ecol Prog Ser. 195: 29-45.

Recibido: 22.04.2019

Aceptado: 21.08.2019 
